\title{
GRAVITATIONAL LENSING STATISTICS BASED ON A LARGE SAMPLE OF HIGHLY LUMINOUS QUASARS ${ }^{1}$
}

\section{J. Surdej² AND J. F. Claeskens}

Institut d'Astrophysique, Université de Liège, Avenue de Cointe 5, B-4000 Liège, Belgium

Electronic mail: surdej@ astra.astro.ulg.ac.be., claeskens@ astra.astro.ulg.ac.be

\section{CRAMPTON}

Dominion Astrophysical Observatory, 5071 West Saanich Road, Victoria, British Columbia V8X 4M6, Canada Electronic mail: crampton@ dao.nrc.ca

\section{A. V. FILIPPENKO}

Department of Astronomy, University of California, Berkeley, California 94720 Electronic mail: alex\%avf.hepnet @ lbl.bitnet

\section{HutSEmÉ Kers AND P. MAGaiN ${ }^{3}$}

Institut d'Astrophysique, Université de Liège, Avenue de Cointe 5, B-4000 Liège, Belgium Electronic mail: hutsemek@ astra.astro.ulg.ac.be, magain @ astra.astro.ulg.ac.be

\section{B. Pirenne}

ST-ECF, c/o ESO, Karl-Schwarzschild str. 2, D-8046 Garching bei München, Germany Electronic mail: bpirenne@eso.org

\section{VANDERRIEST}

Observatoire de Meudon, DAEC, Place J. Janssen 5, F-92195 Meudon Principal Cédex, France Electronic mail: vanderri@frmeu51.bitnet

\section{H. K. C. YEE}

Department of Astronomy, University of Toronto, Toronto, Ontario, Canada M5S 1A7 Electronic mail: hyee@utorphys.bitnet Received 1992 May 15; revised 1992 December 21

\begin{abstract}
Results on gravitational lensing statistics applied to a sample of 469 highly luminous quasars are reported. The objects were directly imaged, either from the ground (ESO, CFH) under optimal seeing conditions, or using the Hubble Space Telescope. We have derived values for the effectiveness parameter $F$ of galaxies, modeled by means of singular isothermal spheres, to produce macrolensed images of distant quasars, and upper limits on the density parameter $\Omega_{L}$ of compact objects with masses $\simeq 10^{10}-10^{12} \mathscr{M} \odot$. Adopting $H_{0}=50 \mathrm{~km} / \mathrm{s} / \mathrm{Mpc}, \Omega_{0}=1$ and $\Lambda=0$, we find that at the $99.7 \%$ confidence level, $0.005<F<0.478$ and that $\Omega_{L}<0.02$. A critical discussion of these results is presented. Finally, comparing the efficiencies of ground-based and space instruments used to search for gravitational lens systems among highly luminous quasars, we conclude that for the near future, ground based direct imaging characterized by a good dynamical range still constitutes the best observational strategy.
\end{abstract}

\section{INTRODUCTION}

Since macrogravitational lens systems provide us with equivalent optical benches having dimensions comparable to that of the Universe, we may conveniently use them in order to infer various parameters of astrophysical (mass of

${ }^{1}$ Based upon observations collected at the European Southern Observatory (La Silla, Chile), with the Canada France Hawaii Telescope and with the Hubble Space Telescope.

${ }^{2}$ Maître de Recherches au Fonds National de la Recherche Scientifique (Belgique) and visiting astronomer at ESO (Garching bei Mūnchen, Germany).

${ }^{3}$ Chercheur Qualifié au Fonds National de la Recherche Scientifique (Belgique). deflecting galaxies, size of intervening gas clouds, etc.) and cosmological ( $H_{0}, \Omega_{0}, \Lambda$, etc.) significance. We refer to Blandford \& Narayan (1992) for a general review on this subject. The possibility of using statistical gravitational lens studies as an astrophysical or cosmological tool has motivated the present work. Indeed, following the theoretical work of Turner et al. (1984), dealing with a realistic determination of the optical depth for macrolensing, it was natural to expect that high angular resolution direct observations of a large sample of bright and distant quasars would constitute the best approach in searching for new lens candidates, and also in setting limits on various astrophysical and cosmological parameters. In the next section, we describe such a sample of 469 highly luminous quasars 
(hereafter HLQs) that have been observed either under good seeing conditions using ground based telescopes at ESO and CFH or with the Hubble Space Telescope (HST).

On the basis of the observed frequency of multiply lensed HLQ images detected in this sample, we present in Sec. 3 results on lensing statistics relevant to (i) the value of the effectiveness parameter $F$ of galaxies to produce multiply lensed quasar images and to (ii) upper limits on the density parameter $\Omega_{L}$ of compact objects with masses $\simeq 10^{10}-10^{12} \mathscr{M}_{\odot}$. Similar statistical results have been recently reported for a sample of 215 quasars and another one of 184 quasars by Surdej et al. (1992b) and Maoz et al. (1992b), respectively.

A discussion as well as general conclusions form the last two sections of the present paper.

\section{DESCRIPTION OF FOUR LARGE SAMPLES OF HIGHLY LUMINOUS QUASARS}

Considering the canonical $\log (N)-B$ relation for the number of quasars per unit area brighter than a given magnitude $B$ (cf. Boyle et al. 1988), it is easy to calculate the number density enhancement of quasars $q\left(M, B_{0}\right)$ in a flux limited sample $B<B_{0}$ subject to a magnification $M$. Following Narayan (1989), one finds that $q\left(M, B_{0}\right)=N\left[<B_{0}\right.$ $+2.5 \log M] /\left(N\left[<B_{0}\right] \times M\right)$. Referring to Fig. 1 of Narayan (1989), where the author has illustrated the dependence of $q$ on both $M$ and $B_{0}$, one immediately sees that the magnification bias becomes more and more important as the limiting flux of the selected sample of quasistellar objects (QSOs) increases. We describe hereafter four major optical surveys for multiply lensed QSO images which benefit from this magnification bias. These QSO samples are subsequently used in Sec. 3 in the context of our statistical gravitational lens studies.

\subsection{The ESO Key Programme Sample}

Following the successful outcomes of the surveys for gravitational lens (GL) candidates among HLQs (typically $\left.M_{V} \leqslant-27 \mathrm{mag}\right)^{2}$ initiated in 1986 November by the Liège/ESO/Hamburg group (Surdej et al. 1988 a,b,c; Swings et al. 1990; Magain et al. 1990) and by Djorgovski \& Meylan (1989a, b), an enlarged team of European and North American astronomers has been successful in obtaining observations at ESO in the framework of a Key Programme (Surdej et al. 1989, 1990, 1992a). Since 1989 May, the Danish $1.5 \mathrm{~m}$ (direct CCD camera), the ESO/ MPI $2.2 \mathrm{~m}$ (direct CCD camera or EFOSC2), the $3.6 \mathrm{~m}$ (EFOSC1) and the NTT (EFOSC2, EMMI or SUSI) telescopes located at La Silla (Chile) have been used to study gravitational lensing effects. The first aim is to detect multiply lensed HLQs with typical angular separations smaller than one arcsec; furthermore, we are also looking for the presence of foreground galaxies in the vicinity of the rele-

${ }^{2}$ Unless quoted otherwise, we have adopted $H_{0}=50 \mathrm{~km} / \mathrm{s} / \mathrm{Mpc}, \Omega_{0}=1$ and $\Lambda=0$. vant targets (cf. Van Drom 1992 and Van Drom et al. 1993 for a more detailed review) and even for the possible signs of a lensing galaxy whose image might be superimposed over that of the objects under study (Magain et al. 1992a; Van Drom et al. 1993). A full detailed account of the direct imagery observations obtained so far at ESO for 187 quasars selected from the Véron-Cetty \& Véron (1987) catalogue may be found in Surdej et al. (1993). This latter compilation lists, for each HLQ observable from the southern hemisphere, its current identification, 1950 equatorial coordinates, redshift, apparent and absolute visual magnitudes (for the latter one, $k$-corrections were applied with $\alpha=-0.7$ and a magnitude correction for the presence of broad emission lines, see Véron-Cetty \& Véron 1991), date of observation, filter and exposure time, seeing (FWHM), types of CCDs and telescopes used, the technique of image analysis that has been applied (direct visualization or point spread function PSF subtraction) and indications on whether the HLQ consists of a confirmed case of a GL (i.e., even if we are pessimistic, we are forced to recognize that it is a GL; for these cases we set $P Q=1$, otherwise $P Q=0$ ) or of just a possible candidate (we are here more optimistic but not totally certain that it is a $\mathrm{GL}$ and we set $O Q=1$, but $P Q=0$, for such candidates). In the latter cases, the distance $\theta_{D Q}$ (in arcsec) and the magnitude difference $M Q$ between the identified secondary point source image and the primary one are also listed. Additional data related to the counts of galaxies near the quasars are also given. Part of this information is summarized in Table 3, which also contains similar data for the three other quasar samples described hereafter.

Accepted cases of gravitationally lensed quasars for which $P Q=1$ (and also of course $O Q=1$ ), identified in the ESO KP sample, have already been reported for the following objects: PG1115+080 (Weymann et al. 1980; this lens has been rediscovered in the course of our survey), UM673 (Surdej et al. 1987) and H1413+117 (Magain et al. 1988). Among the possible GL candidates appearing in the ESO KP sample and for which $O Q=1$ but $P Q=0$, let us mention UM425 (Meylan \& Djorgovski 1989) and Q1208 + 1011 (Magain et al. 1992a,b; Maoz et al. 1992a; Bahcall et al. 1992b). There are, in fact, $18 \mathrm{GL}$ candidates (i.e., with $O Q=1$ and $P Q=0$ ) for which more observations (direct imagery and/or spectroscopy) are required.

Because of the limited dynamical range ( $\Delta m \simeq 5 \mathrm{mag}$ ) of our direct CCD imaging and since galaxies alone are just capable of producing multiply lensed quasars with typical angular separations $\leqslant 3$ " (neglecting thus all possible effects due to clusters of galaxies), we only consider in the remainder those GL candidates for which $\theta_{D Q} \leqslant 3^{\prime \prime}$ and $M Q \leqslant 5$ mag. There remain 17 such candidates characterized by $O Q=1$. Furthermore, because we have been somewhat too generous when selecting optimistic candidates-in order not to lose potential GLs-we estimate that only half of the previous cases (i.e., in total $\simeq 3+(17-3) / 2=10$ ) actually consist of possible GL candidates. 


\subsection{The Crampton et al. Sample}

Using an image stabilizing camera at the CFHT, Crampton et al. (1989) have also made a direct imaging search for closely spaced gravitationally lensed QSO components. The full width at half-maximum intensity (FWHM) of images produced by this camera is found to be typically $10 \%-20 \%$ better than for images recorded with a standard prime focus camera. Out of 32 quasars with $z>1.6$ and $m_{V}<19 \mathrm{mag}$ (i.e., $M_{V}<-26 \mathrm{mag}$ ), seven were found to be possible GL candidates, two of these having subarcsec angular separations. This fraction (22\%) of interesting HLQs is essentially the same as the one (23\%) initially reported by Surdej et al. (1988c) for the Liège/ESO/Hamburg sample. Crampton et al. (1992) have extended their initial survey for lensed quasars to a total sample of 101 HLQs (see Table 3) located north of the equator and extracted from the Véron-Cetty \& Véron (1987) catalogue. Crampton and his collaborators find three quasars for which the residual image remaining after subtraction of the appropriate PSF shows some evidence for additional image(s) with subarcsec separations. In addition, they report 11 quasars which have companion point sources with separations ranging between $\simeq 1$ " and 6". For five of these cases (PKS 0504+03, PG 1715+535, 17449 +206 , PKS $1756+237$ and $2203+29$ ), the companion turns out to be a star. The companion to $4 \mathrm{C} 56.28$ has a redder color than the quasar (Crampton et al. 1989) but we still consider it as a possible GL candidate. There remain six GL candidates for which $O Q=1$, plus the three above with subarcsec separations.

Imposing also here the constraints $\theta_{D Q} \leqslant 3^{\prime \prime}$ and $M Q \leqslant 5$ mag, we are left with just four GL candidates for which $O Q=1$. Again, we estimate that only about half (i.e., $\simeq 2$ ) of these objects constitute possible lens candidates. Additional observations are absolutely mandatory in order to definitely identify their true nature (either $O Q=0$ or $P Q$ $=1$ ). Let us further note that Crampton et al. (1992) report 18 cases of quasars which have nonstellar companions within 6". Therefore, out of 101 observed HLQs, they still identify a significant fraction $(\simeq 32 \%)$ of potentially interesting objects.

\subsection{The Yee et al. Sample}

Yee et al. (1993) report on direct imagery of 104 HLQs obtained in the Gunn $r$ filter with the direct prime focus camera of the CFH telescope (see Table 3). Most of their observations are complementary to those carried out by Crampton et al. (1992). Yee et al. (1993) have rediscovered the gravitational lens $\mathrm{H} 1413+117$ (Magain et al. 1988) and the binary quasar PHL1222 (Djorgovski et al. 1987). Whenever a possible GL candidate was found at the telescope, additional multiband images were obtained. Several possible GL candidates with separation greater than $\simeq 1^{\prime \prime}$ (including PG $1715+535$ and others) have been ruled out this way. For some candidates, spectroscopy has been obtained at Lick Observatory revealing that the close companions are stars. To test for closely-spaced GLs $\left(\leqslant 1^{\prime \prime}\right)$, contour plots of the quasars were compared with reference stars in the field. No such candidates were found. The limits for detection of closely-spaced GLs were derived by simulations. This was done by testing the visibility of artificially added starlike companions with various magnitude differences and at different angular separations (see Yee et al. 1993 for a more detailed account).

\subsection{The HST Snapshot Sample}

A search for gravitationally lensed quasars using the Hubble Space Telescope's Planetary Camera, known as the $H S T$ nonproprietary snapshot survey (Bahcall et al. 1992a), was initiated in 1990 September. It consists in obtaining equivalent $V$ and some $I$ direct images ( 2 or $4 \mathrm{~min}$ and later $260 \mathrm{~s}$ exposures, image scale of $0.043^{\prime \prime} /$ pixel, 4 fields of $\left.34^{\prime \prime} \times 34^{\prime \prime}\right)$, with an angular resolution $\simeq 0.1^{\prime \prime}$, of 354 high luminosity quasars $\left(z>1, M_{V}<-25.5 \mathrm{mag}\right)$ (calculated for $H_{0}=100 \mathrm{~km} / \mathrm{s} / \mathrm{Mpc}, q_{0}=0.5, \alpha=-0.5$ + galactic correction, $|b|>10^{\circ}$ ), selected from the catalogue of Véron-Cetty \& Véron (1987), during gaps in the $H S T$ scheduled observing program. To save time, most of the images are taken using only the gyroscopes for pointing and guiding. Despite the well-known spherical aberration of $H S T$, the existing point spread function still permits the detection of nearby pointlike objects with separations of the order of several tenths of an arcsec. Until the end of 1991 March, useful high-resolution images had been obtained for 32 quasars. Each of these quasar exposures has been examined by eye for evidence of multiple images, the results being calibrated by simulations (Bahcall et al. 1992a). These authors report no evidence of multiple images due to gravitational lensing for these 32 objects. Maoz et al. (1992b) have recently published an additional list of 152 HLQs observed with $H S T$, bringing their total number to 184 quasars. So far, they have only identified one GL candidate, namely the high redshift quasar Q1208+1011 (Maoz et al. 1992a), originally detected from the ground by Magain et al. (1992a) (see also Magain et al. 1992b and Pirenne et al. 1992). Maoz et al. (1992b) identified six other quasars having point sources within 6", and for three of these, ground based observations showed that the companion point sources are foreground galactic stars.

It has been possible for some of us to independently carry out, at the ST-ECF facility (c/o ESO, Garching bei München, Germany), a systematic visual inspection of a sample of PC frames obtained within the HST snapshot survey for 267 quasars (see Table 3), part of these being in the Maoz et al. (1992b) sample. From these observations, we have identified four HLQs whose direct image does show some structure (possibly multiple images-cf. Q1208 + 1011 described in Magain et al. 1992a, b; cases with a superimposed galaxy image, etc.); the known quasarforeground galactic star associations have of course been eliminated from these. Imposing also here the constraints $\theta_{D Q} \leqslant 3^{\prime \prime}$ and $M Q \leqslant 3 \mathrm{mag}$ (because the dynamical range achieved in the $H S T$ snapshot sample is less than that $(M Q \leqslant 5 \mathrm{mag})$ characterizing the ground-based observations, see Sec. 2.5), we still have four GL candidates for which $O Q=1$. We estimate that approximately half of 
TABLE 1. Summary of the observational characteristics of the four selected HLQ samples.

\begin{tabular}{|c|c|c|c|c|c|}
\hline Sample & ESO-KP & Crampton et al. & Yee et al. & HST & Merged sample \\
\hline $\mathrm{N}^{\circ}$ of HLQs & 187 & 101 & 104 & 267 & 469 \\
\hline$<z>$ & 2.3 & 2.4 & 2.2 & 2.2 & 2.2 \\
\hline$\langle V\rangle$ & 17.5 & 18.1 & 17.7 & 17.6 & 17.7 \\
\hline$<M_{V}>$ & 28.0 & -27.5 & -27.8 & -27.8 & -27.7 \\
\hline$<F W H M>$ & $1.05 "$ & $0.67^{\prime \prime}$ & $0.76^{\prime \prime}$ & & \\
\hline \multicolumn{6}{|c|}{$N^{\circ}$ of HLQs with } \\
\hline$(\alpha)$ & 3 & 0 & 1 & 0 & 3 \\
\hline (3) & 21 & 9 & 2 & 4 & 31 \\
\hline$(\gamma)$ & 17 & 4 & 1 & & \\
\hline & & & & & 23 \\
\hline$(\delta)$ & & & & 4 & \\
\hline $\begin{array}{l}\text { Most probable } \\
\mathrm{N}^{\circ} \text { of HLQs }\end{array}$ & & & & & \\
\hline with $O Q=1$ & 10 & 2 & 1 & 2 & 13 \\
\hline
\end{tabular}

\section{( $\alpha) P Q=1$}

(及) $\mathrm{OQ}=1$

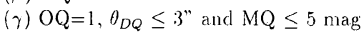

( $\delta) O Q=1.0_{D Q} \leq 3 "$ and $M Q \leq 3 \mathrm{mag}$.

these interesting HLQs (i.e., $\simeq 2$ ) constitute new possible GL candidates. Let us note that we did not identify in this sample any new, yet confirmed, gravitational lens (i.e., for which $P Q=1$ ).

\subsection{Merging the Four HLQ Samples}

The most important observational characteristics of the four HLQ samples described above are listed in Table 3 (totaling 659 observations; see Surdej et al. 1993, Crampton et al. 1992, Yee et al. 1993, Bahcall et al. 1992a, and

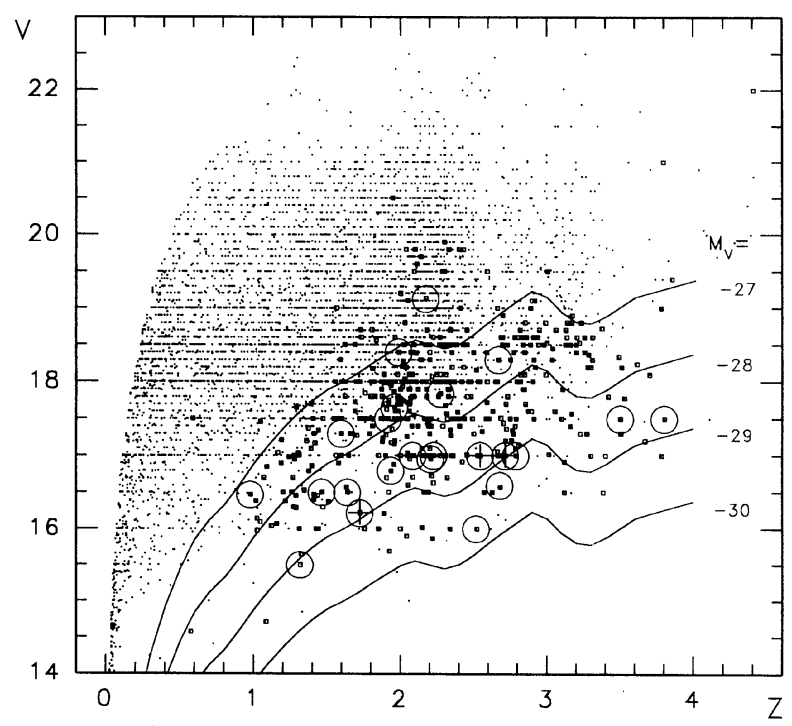

FIG. 1. Apparent magnitude ( $V$ ) vs redshift ( $z$ ) diagram showing the locations of 6003 quasars (very small dots) extracted from the VéronCetty \& Véron (1991) catalogue. Note that not all apparent magnitudes quoted for the quasars in the above catalogue are visual and that most of them are just estimates. The 469 selected HLQs are shown by small squares, the 23 (although only about half of these probably consist of) possible lens candidates $(O Q=1)$ by circles, and the three known lensed quasars $(P Q=1)$ identified in the merged sample by crosses. Several lines of constant absolute visual magnitude are also shown.
TABLE 2. Adopted "angle selection functions" (ASFs).
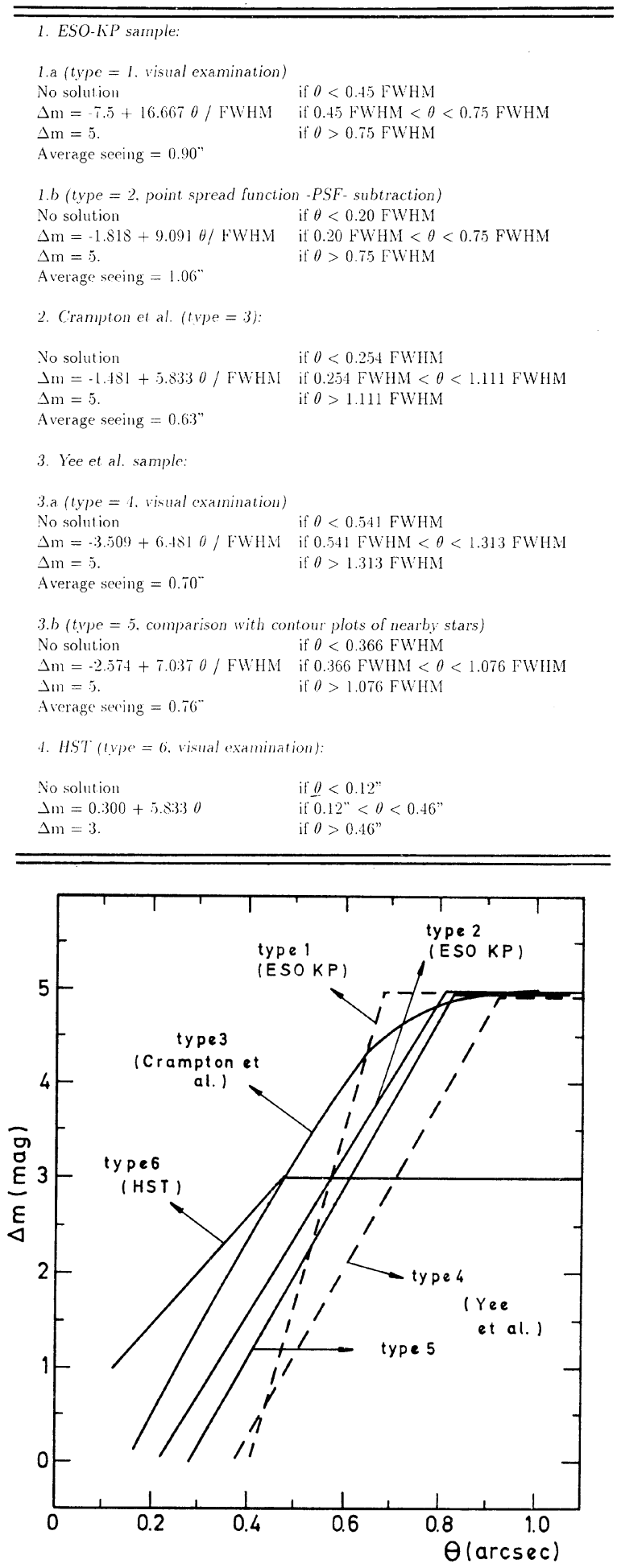

FIG. 2. The "angle selection functions" (ASFs), derived under average seeing conditions (see $\langle F W H M\rangle$ in Table 1), for the various types of ground based (Type $=1-5$, see Table 2) and $H S T$ ( Type $=6$ ) observations. The maximum detectable magnitude difference $\Delta m$ between two images is plotted here as a function of their separation $\theta$. Whereas the $H S T$ angle selection function is somewhat better for $\theta \leqslant 0.4$ " (equivalent to ground based seeing conditions near $0.7^{\prime \prime}$ ), the better dynamical range of ground based CCDs accounts for better ASFs when $\theta \geqslant 0.4^{\prime \prime}$. 
Maoz et al. 1992b for more details). A summary of the observational characteristics of each sample is given in Table 1 and is also partly illustrated in Fig. 1.

Defining the "angle selection function" (ASF) as being the maximum detectable magnitude difference $\Delta m$ between two pointlike images separated by an angle $\theta$, we have listed in Table 2 these ASFs for each of the four HLQ samples and the various methods of image analysis that have been used: i.e., "Type" $=1$ (ESO-KP, visual examination), 2 (ESO-KP, PSF subtraction), 3 (Crampton et al.), 4 (Yee et al., visual examination), 5 (Yee et al., comparison with contour plots of nearby stars), 6 (HST, visual examination); see also Fig. 2.

Statistical estimates of the optical depth for gravitational macrolensing based upon the data merged from these four individual HLQ samples are reported in the next section. Note that because of observational duplication, there remains a total of 469 distinct quasars (those observations which turned out to be the best ones for the calculation of the effectiveness parameter $F$ in Sec. 3 are indicated with an * in Table 3 ). In this merged sample of HLQs, we finally adopt $3 \leqslant N_{L} \leqslant 13$ for the observed number $N_{L}$ of lenses. The lower limit corresponds to the "pessimistic" number of lenses present in the merged sample, and the upper limit to the "optimistic" number.

\section{GRAVITATIONAL LENSING STATISTICS:}

\subsection{The Effectiveness Parameter F of Galaxy Lenses}

\subsubsection{The method}

Following Turner et al. (1984, hereafter TOG) and, more recently, Nemiroff (1989), Fukugita \& Turner (1991, hereafter FT), Kochanek (1991a), Mao (1991), and Schneider (1991), the effective optical depth $\tau$ of galaxies (modeled by singular isothermal spheres) to produce multiple macrolensed images of distant quasars may be expressed as

$$
\begin{aligned}
\tau=N_{L} / N_{Q}= & F \sum_{q=1}^{N_{Q}} f\left(z_{q}\right) B\left(b_{q}, \Delta_{\min }\right) P\left(>\theta_{q}\right) \\
& \times\left[\frac{10^{\Delta m / 2.5}-1}{10^{\Delta m / 2.5}+1}\right]^{2} S_{\mathrm{cat}} / N_{Q},
\end{aligned}
$$

where

$-N_{L}$ represents the number of multiply imaged objects expected within a sample of $N_{Q}$ quasars with redshifts $z_{q}$, blue apparent magnitudes $b_{q}$, and having been imaged under an equivalent (atmospheric or instrumental) angular resolution $\theta_{q}$. Note that for all the HLQs listed in Table 3, we have derived their $b_{q}$ magnitudes from their $V$ magnitudes tabulated in the Véron-Cetty \& Véron catalogue (1991) by applying an average $B-V$ correction as a function of their redshift $z_{q}$. Once more, we stress here that not all apparent magnitudes quoted for the quasars in the above catalogue are "visual" and that most of these values just constitute rough estimates.

$-F \propto n_{0} \sigma^{4}$ measures the effectiveness of cosmically distributed "singular isothermal spherical" (SIS) galaxies to produce double QSO images ( $n_{0}$ being the local number density and $\sigma$ the one-component velocity dispersion of those galaxies, see TOG). From observed parameters of local galaxies and adopting an SIS model, FT, Kochanek (1991b) and Mao (1991) have calculated that $F \simeq 0.047$ \pm 0.011 , with $90 \%$ contributed by $\mathrm{E}$ and S0 galaxies. All these authors also report that $0.023 \leqslant F \leqslant 0.047$ when the effects of a finite core radius are taken into account. Note that these estimates are independent of the value adopted for $H_{0}$ and that FT actually confirm that the core radii of $\mathrm{E}$ and $\mathrm{SO}$ galaxies seem to be very small;

$-f\left(z_{Q}\right)$ accounts for the redshift dependence of the optical depth, i.e., following TOG:

$$
\begin{gathered}
f\left(z_{q}\right)=\frac{\left[\left(y^{4}+4 y^{2}+1\right) \ln (y)-3 / 2\left(y^{4}-1\right)\right]}{4\left(y^{2}-1\right)^{2}} \\
\text { for } \Omega_{0}=0, \quad \text { (empty Universe) }
\end{gathered}
$$

and

$f\left(z_{q}\right)=\frac{4\left(y^{1 / 2}-1\right)^{3}}{15 y^{3 / 2}}$ for $\Omega_{0}=1$ (Einstein-de Sitter),

where $y=\left(1+z_{q}\right)$,

$-B\left(b_{q}, \Delta_{\min }\right)$ corrects for the amplification-or more appropriately, for the magnification-bias (cf. Sec. 1). For a given redshift, the magnification bias results in the observed counts of quasars being given by the convolution of the intrinsic luminosity function with a magnification probability distribution. Adopting the quasar counts $n_{Q}\left(b_{q}\right)$ reported by Hartwick \& Schade (1990) and the generalized point source amplification probability $P\left(\Delta, \Delta_{\text {min }}\right)$ derived from the SIS lens model

$$
P\left(\Delta, \Delta_{\min }\right)=1.84210^{0.8\left(\Delta_{\min }-\Delta\right)},
$$

which is such that $\int_{\Delta_{\min }}^{\infty} P\left(\Delta, \Delta_{\min }\right) d \Delta=1$, it is straightforward to derive the following expression for the magnification bias

$$
B\left(b_{q}, \Delta_{\min }\right)=\frac{\int_{\Delta_{\min }}^{\infty} n_{Q}\left(b_{q}+\Delta\right) P\left(\Delta, \Delta_{\min }\right) d \Delta}{n_{Q}\left(b_{q}\right)},
$$

for the particular value of the minimum magnitude amplification $\Delta_{\mathrm{min}}$. Let us note that if we were able to resolve two lensed images characterized by an infinite brightness ratio, we would have $\Delta_{\min }=2.5 \log (2)$ (see TOG). However, because of the finite dynamical range of current imaging instruments, we have $\Delta_{\min }>2.5 \log (2)$.

-the correction function $P\left(>\theta_{q}\right)$ represents the fraction of expected lenses with multiple image separations greater than the angular resolution $\theta_{q}$ used for observing the particular quasar(s). On the basis of numerical simulations and considering observations carried out from the ground, under optimal seeing conditions, FT have estimated that, typically, $P\left(>\theta_{q}\right) \simeq 50 \%-90 \%$. Note that the function $P\left(>\theta_{q}\right)$ derived by FT does not depend on $H_{0}$, nor on $z_{q}$,

-the factor appearing between the brackets in Eq. (1) accounts for our inability to detect two lensed quasar im- 
ages whose magnitude difference is greater than $\Delta m$. Physically, it merely accounts for the reduction in number of potential SIS lenses located along our line of sight that are capable of producing detectable multiply lensed images. Of course, the larger the instrumental dynamical range $\Delta m$, the better it is for searching for new lenses,

-finally, because of criteria imposed on the image morphology, color, proper motion, etc. while selecting quasar candidates, existing quasar catalogues are thought to be biased against the inclusion of gravitationally lensed objects. Indeed, quasar candidates might have been excluded from quasar catalogues because they appear either not to be stellarlike (e.g., made of multiple images), or somewhat redder than expected (because of a possible contamination by a deflecting galaxy) or even, possibly affected by an apparent proper motion (induced by light variability of one or more of its components). Taking into account the two first observational biases, Kochanek (1991b) has estimated that a loss of up to $30 \%$ of GL systems may result. Therefore, in order to correct for these biases, we have set $S_{\text {cat }} \simeq 0.7$ in Eq. (1).

When deriving the above expression for the effective optical depth $\tau$, TOG and FT have explicitly assumed that our ability to resolve two pointlike images in the angular range $\left[\theta_{\text {res }}, \theta_{\max }\right]$ is characterized by a constant dynamical range $\Delta m$. This assumption naturally accounts for the presence of the factor $P\left(>\theta_{q}\right)\left[\left(10^{\Delta m / 2.5}-1\right) /\left(10^{\Delta m / 2.5}\right.\right.$ $+1)]^{2}$ in Eq. (1). In our particular case, we know that the dynamical range $\Delta m$ is actually a function of the angular separation $\theta$ [i.e., $\Delta m=\Delta m(\theta)$; see Table 2 and Fig. 2]. We thus have to correct Eq. (1) for this dependence and write

$$
\tau=N_{L} / N_{Q}=F \sum_{q=1}^{N_{Q}} f\left(z_{q}\right) H\left(b_{q}, \text { Type, } \theta_{\max }\right) S_{\text {cat }} / N_{Q},
$$

where the function

$$
\begin{aligned}
& H\left(b_{q}, \text { Type }, \theta_{\max }\right) \\
& =\frac{\int_{0}^{\theta_{\max }} p(\theta) B\left(b_{q}, \Delta_{\min }(\theta)\right)\left[\frac{10^{\Delta m(\theta) / 2.5}-1}{10^{\Delta m(\theta) / 2.5}+1}\right]^{2} d \theta}{\int_{0}^{\infty} p(\theta) d \theta}
\end{aligned}
$$

depends of course on the particular set of observations considered (via the expression of the ASFs given in Table 2 for the different values of Type $=1-6$ ) and on the maximum angular radius $\theta_{\max }$ (set here to $3^{\prime \prime}$ ) of the field under consideration. The quantity $p(\theta) d \theta$ represents the probability for the angular separation between two source images produced by an SIS lens model to lie in the range $[\theta, \theta+d \theta]$ (see the form of the function $p(\theta)$ given by FT). Furthermore, because of the limited instrumental dynamical range $\Delta m(\theta)$ that is available to resolve multiply lensed images, the argument $\Delta_{\min }(\theta)$ appearing in the above expression of the magnification bias must also be changed and, for the particular SIS lens model, be set equal to

$$
\begin{aligned}
\Delta_{\min }(\theta)= & 2.5 \log \left[\frac{2\left(10^{\Delta m(\theta) / 2.5}+1\right)}{\left(10^{\Delta m(\theta) / 2.5}-1\right)}\right] \\
& \text { 3.1.2 Numerical applications and results }
\end{aligned}
$$

We consider together the ESO-KP, Crampton et al, Yee et al., and HST snapshot samples of $469=(187 \mathrm{U} 101$ U 104 U 267) HLQs (see Table 3), for which we have also listed in Table 1 the numbers $N_{L}^{O}$ (optimistic estimate) and $N_{L}^{P}$ (pessimistic estimate) of possible lens candidates and known cases of gravitational lensing, respectively. We have then derived by means of Eq. (6) lower and upper limits for the effectiveness parameter $F$, taking into account the dependence of the functions $f\left(z_{q}\right)$ and $H\left(B_{q}\right.$,Type, $\left.\theta_{\max }\right)$ for each HLQ, assuming various types of cosmologies (cf. different values of the normalized cosmological parameter $\lambda_{0}=\Lambda / 3 H_{0}^{2}$ ). Conversely, we have adopted the range of values $0.023<F<0.047$, inferred by FT for the effectiveness parameter $F$ of SIS galaxies, and we have derived upper $\left(N_{\mathrm{up}}^{\exp }\right)$ and lower $\left(N_{\mathrm{lo}}^{\mathrm{exp}}\right)$ estimates for the expected numbers of lenses. Our results are summarized in Table 4.

\subsubsection{Discussion}

For the case of an Einstein-de Sitter cosmology $\left(\Omega_{0}=1\right.$, $\Lambda=0$ ), we find that, at a $99.7 \%$ confidence level, $0.005<F$ $<0.478$. This large interval of values encompasses the predicted value $F=0.047 \pm 0.011$ for the case of a singular isothermal sphere and $0.023 \leqslant F \leqslant 0.047$ when the effects of a finite core radius are taken into account (see FT, Kochanek 1991b, and Mao 1991). Furthermore, our statistical results seem to confirm the claims by Turner (1990), Fukugita et al. (1990, hereafter FFK), Fukugita et al. (1992, hereafter FFKT), FT and others that flat universe models excessively dominated by the cosmological constant are not favored. However, due to a number of complications discussed hereafter, the possibility of estimating the cosmological parameter $\Lambda$ from the observed rate of quasars being lensed by galaxies should be considered presently with great caution.

Indeed, whereas the optical depth $\tau$ is quite sensitive to the cosmological parameter $\Lambda$ (cf. Turner 1990, FFK, Mao 1991, FFKT, FT, Kochanek 1991c), it also depends very strongly on various other factors. For instance:

(i) $\tau$ depends on $\Omega_{0}$ as well as on the assumed (large) degree of homogeneity of the Universe;

(ii) $\tau$ depends on the adopted distribution of galaxy types, on the exact form of the velocity dispersion, which is itself closely related to the (Schechter) luminosity function as well as on the accuracy and applicability of the observed luminosity-velocity dispersion (Faber-Jackson) or rotation curve (Tully-Fischer) relations. It also depends on the implicit existence of massive dark halos around elliptical galaxies (for which $\sigma_{\text {eff }}=\sigma \sqrt{3 / 2}$ and on the adopted mass distribution $\left(\rho \propto 1 / r^{2}\right)$ for elliptical, spiral, etc. galaxies;

(iii) following Kochanek (1991b), the optical depth $\tau$ could also be quite sensitive to reddening effects induced by the lensing galaxies, if they are significant at all; 
TABLE 3. Observational characteristics of the HLQs (see notes below).

\begin{tabular}{|c|c|c|c|c|c|c|c|c|c|c|c|}
\hline Identif & fication & F & Tyре & $z$ & $\mathrm{~m}$ & M & FWHM & $P Q$ & $\mathrm{OQ}$ & $\theta_{D Q}$ & MQ \\
\hline$Q$ & $0002-122$ & * & 1 & 2.758 & 17.21 & -28.8 & 0.65 & 0 & 0 & & \\
\hline UM & 197 & & 4 & 2.180 & 18.70 & -26.7 & 0.78 & 0 & 0 & & \\
\hline UM & 197 & & 1 & 2.180 & 18.00 & -27.4 & 1.15 & 0 & 0 & & \\
\hline UM & 197 & & 6 & 2.180 & 18.00 & -27.4 & & 0 & 0 & & \\
\hline UM & 197 & * & 3 & 2.180 & 18.00 & -27.4 & 0.90 & 0 & 0 & & \\
\hline UM & 18 & * & 1 & 1.890 & 16.21 & -29.1 & 0.70 & 0 & 0 & & \\
\hline UM & 18 & & 5 & 1.890 & 16.21 & -29.1 & 0.86 & 0 & 0 & & \\
\hline UM & 18 & & 6 & 1.890 & 16.21 & -29.1 & & 0 & 0 & & \\
\hline TEX & $0004+171$ & * & 6 & 2.890 & 18.50 & -27.7 & & 0 & 0 & & \\
\hline PKS & $0005-239$ & * & 1 & 1.410 & 16.47 & -28.2 & 0.67 & 0 & 0 & & \\
\hline Q & $0007-4239$ & x & 1 & 2.670 & 18.30 & -27.6 & 0.94 & 0 & 1 & 2.4 & 4.5 \\
\hline$Q$ & $000 \bar{\tau}-35+t$ & * & 6 & 2.030 & 18.04 & -27.4 & & 0 & 0 & & \\
\hline UM & 208 & & 4 & 2.310 & 18.50 & -27.2 & 0.66 & 0 & 0 & & \\
\hline UM & 208 & & 1 & 2.310 & 17.00 & -28.7 & 0.72 & 0 & 0 & & \\
\hline UM & 208 & & 6 & 2.310 & 17.00 & -28.7 & & 0 & 0 & & \\
\hline UM & 208 & * & 3 & 2.310 & 17.00 & -28.7 & 0.57 & 0 & 0 & & \\
\hline UM & 209 & × & 1 & 1.560 & 17.00 & -27.9 & 0.53 & 0 & 0 & & \\
\hline Plis & $0008+171$ & * & 4 & 1.601 & 18.00 & -26.9 & 0.75 & 0 & 0 & & \\
\hline UM & 211 & * & 3 & 2.000 & 16.00 & -29.4 & 0.63 & 0 & 0 & & \\
\hline $\mathrm{Q}$ & $0011-012$ & * & 1 & 2.230 & 17.00 & -28.4 & 1.00 & 0 & 1 & 3.0 & 2.0 \\
\hline UM & $2 \cdot 2 \cdot 4$ & * & 1 & 2.086 & 17.00 & -28.5 & 0.62 & 0 & 1 & 0.0 & 0.0 \\
\hline UM & 224 & & 6 & 2.086 & 17.00 & -28.5 & & 0 & 0 & & \\
\hline UM & 224 & & 4 & 2.086 & 18.20 & -27.3 & 0.66 & 0 & 1 & 3.3 & 5.4 \\
\hline UM & 224 & & 3 & 2.090 & 17.00 & -28.5 & 0.65 & 0 & 1 & 3.2 & 5.6 \\
\hline S5 & $0014+81$ & & 6 & 3.384 & 16.50 & -29.3 & & 0 & 0 & & \\
\hline S5 & $0014+81$ & * & 3 & 3.384 & 16.50 & -29.3 & 0.70 & 0 & 0 & & \\
\hline $3 \mathrm{C}$ & 9.0 & * & 5 & 2.012 & 18.21 & -27.2 & 0.66 & 0 & 0 & & \\
\hline Q & $0018-422$ & & 1 & 2.860 & 18.60 & -27.6 & 1.12 & 0 & 0 & & \\
\hline Q & $0018-122$ & $*$ & 6 & 2.860 & 18.60 & -27.6 & & 0 & 0 & & \\
\hline UM & 6633 & * & 6 & 2.000 & 17.90 & .27 .5 & & 0 & 0 & & \\
\hline UM & 30 & & 6 & 2.050 & 19.10 & -26.3 & & 0 & 0 & & \\
\hline $\mathrm{UN}$ & 30 & * & 3 & 2.050 & 18.00 & $2 \pi .4$ & 0.55 & 0 & 0 & & \\
\hline $\mathrm{WM}$ & 35 & & ). & 2.420 & 18.00 & -27.8 & 0.66 & 0 & 0 & & \\
\hline $\mathrm{UM}$ & 3.5 & * & 3 & 2.420 & 18.00 & -27.8 & 0.68 & 0 & 0 & & \\
\hline $\mathrm{UN}$ & 2.47 & * & 3 & 2.350 & 17.00 & -28.7 & 0.60 & 0 & 0 & & \\
\hline $\mathrm{IM}$ & 42 & * & 3 & 2.260 & 18.00 & -27.4 & 0.67 & 0 & 0 & & \\
\hline UM & 252 & * & 4 & 1.732 & 18.60 & -26.5 & 0.68 & 0 & 0 & & \\
\hline $\mathrm{COM}$ & 253 & * & 2 & 2.222 & 18.60 & -26.8 & 0.72 & 0 & 0 & & \\
\hline $1 \mathrm{MI}$ & 2.53 & & 4 & 2.222 & 18.60 & -26.8 & 0.70 & 0 & 0 & & \\
\hline 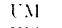 & $6(6.5$ & & 6 & 2.650 & 18.00 & 27.8 & & 0 & 0 & & \\
\hline $\mathrm{CM}$ & 66.5 & * & + & 2.650 & 18.00 & $-2 \pi .8$ & 0.70 & 0 & 0 & & \\
\hline & $00 \cdot 29+0722$ & * & 5 & 3.259 & 18.40 & -27.3 & 0.58 & 0 & 0 & & \\
\hline & $00: 9+07 \cdot 2$ & & 6 & 3.259 & 18.10 & .27 .3 & & 0 & 0 & & \\
\hline & $0029+0722$ & & 3 & 3.272 & 18.40 & .27 .3 & 0.75 & 0 & 0 & & \\
\hline UM & 45 & * & 1 & 1.990 & 18.40 & -27.0 & 0.71 & 0 & 1 & 0.0 & 0.0 \\
\hline UM & 46 & * & 3 & 2.310 & 18.00 & -27.7 & 0.46 & 0 & 0 & & \\
\hline UM & 259 & * & 1 & 1.850 & 17.00 & -28.2 & 0.77 & 0 & 0 & & \\
\hline UM & 259 & & 4 & 1.850 & 17.00 & -28.2 & 0.69 & 0 & 0 & & \\
\hline $4 \mathrm{C}$ & 09.01 & * & 1 & 1.918 & 17.50 & -27.8 & 0.89 & 0 & 1 & 0.0 & 0.0 \\
\hline $4 \mathrm{C}$ & 09.01 & & 6 & 1.918 & 17.50 & .27 .8 & & 0 & 0 & & \\
\hline $4 \mathrm{C}$ & 09.01 & & 4 & 1.918 & 17.50 & -27.8 & 1.22 & 0 & 0 & & \\
\hline$Q$ & $0034-3308$ & * & 1 & 2.180 & 17.80 & -27.6 & 1.06 & 0 & 0 & & \\
\hline UM & 52 & ${ }^{*}$ & 4 & 2.270 & 18.00 & -27.4 & 0.67 & 0 & 0 & & \\
\hline $5 \mathrm{C}$ & 03.44 & * & 6 & $\Gamma .937$ & 17.95 & -27.4 & & 0 & 0 & & \\
\hline $\mathrm{Q}$ & $0038-3936$ & * & 6 & 2.370 & 18.00 & .27 .8 & & 0 & 0 & & \\
\hline $\mathrm{CT}$ & 1.5 & * & 1 & 1.803 & 17.50 & -27.7 & 0.75 & 0 & 0 & & \\
\hline$Q$ & $0039-26.5$ & * & 6 & 1.803 & 17.50 & $-2 \pi .7$ & & 0 & 0 & & \\
\hline & $0041-2707$ & * & 1 & 2.78 .3 & 17.40 & -28.6 & 0.69 & 0 & 0 & & \\
\hline Q & $0041-261$ & * & 1 & 2.501 & 17.30 & -28.3 & 0.96 & 0 & 0 & & \\
\hline & $0042-2627$ & * & 1 & 3.400 & 17.00 & -28.8 & 0.74 & 0 & 0 & & \\
\hline UM & 275 & * & 1 & 2.150 & 17.00 & -28.4 & 0.64 & 0 & 0 & & \\
\hline UM & 275 & & 6 & 2.150 & 17.00 & .28 .4 & & 0 & 0 & & \\
\hline UM & 275 & & 4 & 2.150 & 17.00 & -28.4 & 1.34 & 0 & 0 & & \\
\hline LiM & 275 & & 3 & 2.150 & 17.00 & -28.4 & 0.66 & 0 & 0 & & \\
\hline $\mathrm{LM}$ & $2 \pi 6$ & * & 6 & 1.590 & 18.30 & .26 .6 & & 0 & 0 & & \\
\hline UN & $66 \mathrm{~T}$ & & 6 & 3.135 & 18.60 & .27 .2 & & 0 & 0 & & \\
\hline UM & 667 & * & 3 & 3.140 & 18.60 & -27.2 & 0.88 & 0 & 0 & & \\
\hline $\mathrm{UM}$ & 278 & & 6 & 2.530 & 18.00 & .27 .6 & & 0 & 0 & & \\
\hline UM & 278 & * & 4 & 2.5 .30 & 18.00 & .27 .6 & 0.52 & 0 & 0 & & \\
\hline UM & 278 & & 3 & 2.530 & 18.00 & 27.6 & 0.76 & 0 & 0 & & \\
\hline PKS & $0046-06$ & * & 5 & 2.060 & 18.00 & -27.5 & 0.52 & 0 & 0 & & \\
\hline PKS & $0046-315$ & * & 6 & 2.721 & 17.70 & -28.2 & & 0 & 0 & & \\
\hline UM & 281 & * & 1 & $1.8 \pi 0$ & 17.90 & -27.4 & 0.66 & 0 & 0 & & \\
\hline $1 \mathrm{M}$ & 281 & & 6 & 1.870 & 17.90 & -27.4 & & 0 & 0 & & \\
\hline$Q$ & $00 \mid 8-261$ & * & 6 & 2.249 & 18.10 & -27.3 & & 0 & 0 & & \\
\hline Cs & 73 & * & 6 & $2.2 \pi 2$ & 18.10 & .27 .3 & & 0 & 0 & & \\
\hline UMI & 287 & * & 1 & 2.270 & 17.00 & -28.4 & 0.72 & 0 & 0 & & \\
\hline UM & 287 & & 6 & 2.270 & 17.00 & -28.4 & & 0 & 0 & & \\
\hline UM & 287 & & + & 2.270 & 17.00 & -28.4 & 0.73 & 0 & 0 & & \\
\hline$Q$ & $0049-393$ & * & 6 & 2.845 & 17.90 & -28.2 & & 0 & 0 & & \\
\hline UM & 288 & * & 1 & 2.310 & 17.00 & -28.7 & 0.68 & 0 & 0 & & \\
\hline UM & 288 & & 5 & 2.310 & 17.00 & -28.7 & 0.68 & 0 & 0 & & \\
\hline$U M$ & 288 & & 6 & 2.310 & 17.00 & -28.7 & & 0 & 0 & & \\
\hline $1 \mathrm{M}$ & 288 & & 3 & 2.310 & 17.00 & -28.7 & 0.75 & 0 & 0 & & \\
\hline B2 & $00.51+29$ & * & 6 & 1.828 & 17.80 & -27.4 & & 0 & 0 & & \\
\hline$Q$ & $0052-4104$ & * & 6 & 2.060 & 18.10 & 27.4 & & 0 & 0 & & \\
\hline
\end{tabular}


TABLE 3. (continued)

\begin{tabular}{|c|c|c|c|c|c|c|c|c|c|c|c|}
\hline Identif & fication & $\mathrm{F}$ & Type & $\mathrm{z}$ & $\mathrm{m}$ & $\mathrm{M}$ & FWHM & $\mathrm{PQ}$ & $\mathrm{OQ}$ & $\theta_{D Q}$ & $\mathrm{MQ}$ \\
\hline UM & 375 & * & 3 & 2.020 & 18.00 & -27.4 & 0.66 & 0 & 0 & & \\
\hline PHL & 1222 & & 6 & 1.904 & 17.63 & -27.7 & & 0 & 0 & & \\
\hline PHL & 1222 & * & 4 & 1.904 & 17.63 & -27.7 & 0.65 & 0 & 0 & & \\
\hline S5 & $0153+74$ & & 6 & 2.338 & 16.00 & -29.8 & & 0 & 0 & & \\
\hline S5 & $0153+\pi 1$ & * & 3 & 2.338 & 16.00 & -29.8 & 0.88 & 0 & 0 & & \\
\hline UM & 148 & * & 1 & 2.991 & 18.80 & -27.3 & 0.62 & 0 & 0 & & \\
\hline & $0154-512 \mathrm{C}$ & * & 6 & 1.660 & 17.30 & $-27 . \bar{T}$ & & 0 & 0 & & \\
\hline UM & 154 & * & 5 & 2.432 & 18.20 & -27.7 & 0.63 & 0 & 0 & & \\
\hline B2 & $0201+36 \mathrm{~B}$ & * & 6 & 2912 & 17.50 & -28.7 & & 0 & 0 & & \\
\hline & $0203-498$ & * & 2 & 2.540 & 18.00 & -27.7 & 1.42 & 0 & 0 & & \\
\hline$Q$ & $0205-379$ & $*$ & 6 & 2.404 & 17.40 & -28.1 & & 0 & 0 & & \\
\hline UM & 400 & * & 6 & 1.890 & 18.10 & -27.2 & & 0 & 0 & & \\
\hline UM & 402 & & 1 & 2.856 & 17.70 & -28.4 & $1.0 \bar{i}$ & 0 & 0 & & \\
\hline UM & 402 & & 4 & 2.856 & 17.70 & -28.4 & 0.69 & 0 & 0 & & \\
\hline $\mathrm{UM}$ & 402 & * & 3 & 2.840 & 16.00 & -30.1 & 0.55 & 0 & 0 & & \\
\hline UM & $40: 3$ & & 1 & 2.190 & 18.00 & -27.4 & 1.57 & 0 & 0 & & \\
\hline UM & 403 & & +4 & 2.190 & 18.00 & $\begin{array}{l}-27.4 \\
-27.4\end{array}$ & 0.65 & 0 & 0 & & \\
\hline $\mathrm{UM}$ & 403 & $*$ & 3 & 2.190 & 18.00 & -27.4 & 0.89 & 0 & 0 & & \\
\hline Q & $0207-398$ & $*$ & 2 & 2.805 & 17.15 & -28.9 & 1.21 & 0 & 0 & & \\
\hline Q & $0207-398$ & & 6 & 2.80 .5 & 17.15 & -28.9 & & 0 & 0 & & \\
\hline TEX & $0215+16.5$ & * & 6 & 1.900 & 18.00 & -27.3 & & 0 & 0 & & \\
\hline & $0216+0803$ & & 6 & 2.993 & 18.10 & -28.0 & & 0 & 0 & & \\
\hline & $0216+0803$ & & 4 & 2.993 & 18.10 & -28.0 & 0.72 & 0 & 0 & & \\
\hline & $0216+0803$ & × & 3 & 2.991 & 18.10 & .28 .0 & 0.53 & 0 & 0 & & \\
\hline Q & $0222-41.5$ & & $i$ & 2.000 & 17.70 & -27.7 & 1.34 & 0 & 0 & & \\
\hline Q & $0222-41.5$ & * & 6 & 2.000 & 17.70 & -27.7 & & 0 & 0 & & \\
\hline PKS & $0225-014$ & $*$ & 3 & 2.037 & 18.20 & -27.2 & 0.48 & 0 & 0 & & \\
\hline PKS & $0226-038$ & * & 5 & 2.064 & 16.96 & -28.5 & 0.70 & 0 & 0 & & \\
\hline PKS & $0226-0.38$ & & 6 & 2.064 & 16.96 & -28.5 & & 0 & 0 & & \\
\hline PKS & $0229+13$ & & 6 & 2.065 & 17.71 & -27.8 & & 0 & 0 & & \\
\hline PKS & $0229+13$ & & 4 & 2.065 & 17.71 & -27.8 & 0.64 & 0 & 0 & & \\
\hline PKS & $0229+13$ & * & 3 & 2.065 & 17.70 & -27.8 & 0.67 & 0 & 0 & & \\
\hline PIS & $0232-0.4$ & * & 1 & 1.434 & 16.46 & -28.3 & 0.75 & 0 & 0 & & \\
\hline PLS & $0232-0.1$ & & 6 & 1.43 .4 & 16.16 & -28.3 & & 0 & 0 & & \\
\hline PLS & $0231-301$ & ${ }^{*}$ & 2 & 2.102 & 18.00 & -27.5 & 1.46 & 0 & 0 & & \\
\hline PKS & $0237-23$ & * & 1 & 2.224 & 16.63 & -28.8 & 0.71 & 0 & 0 & & \\
\hline UM & 677 & & 1 & 2.782 & 18.60 & -27.4 & 1.74 & 0 & 0 & & \\
\hline UM & $6 \pi 7$ & * & 6 & 2.782 & 18.60 & -27.4 & & 0 & 0 & & \\
\hline PKS & $0244-128$ & * & 1 & 2.201 & 17.10 & -28.3 & 0.58 & 0 & 0 & & \\
\hline PKS & $0244-128$ & & 6 & 2.201 & 17.10 & -28.3 & & 0 & 0 & & \\
\hline PKS & $0244-128$ & & 4 & 2.201 & 17.10 & -28.3 & 0.70 & 0 & 0 & & \\
\hline S4 & $0248+43$ & * & 6 & 1.316 & 15.50 & -29.0 & & 0 & 1 & 0.6 & 2.5 \\
\hline$U \mathrm{M}$ & 678 & & 2 & 3.202 & 18.40 & -27.3 & 1.66 & 0 & 0 & & \\
\hline UM & 678 & * & 6 & 3.202 & 18.40 & -27.3 & & 0 & 0 & & \\
\hline UM & 679 & & 2 & 3.205 & 18.60 & -27.1 & 1.66 & 0 & 0 & & \\
\hline UM & 679 & * & 6 & 3.205 & 18.60 & -27.1 & & 0 & 0 & & \\
\hline$Q$ & $0254-404$ & $*$ & 2 & 2.290 & 17.40 & -27.6 & 1.19 & 0 & 0 & & \\
\hline Q & 0254-404 & & 6 & 2.280 & 17.40 & .27 .9 & & 0 & 0 & & \\
\hline Q & $0254-334$ & * & 1 & 1.849 & 16.00 & -29.2 & 0.66 & 0 & 0 & & \\
\hline Q & $0256-0000$ & $*$ & 5 & 3.374 & 18.72 & -27.1 & 0.64 & 0 & 0 & & \\
\hline Q & 0256-0000 & & 3 & 3.367 & 18.70 & -27.1 & 0.75 & 0 & 0 & & \\
\hline$Q$ & $0301-0035$ & & 2 & 3.223 & 18.53 & -27.2 & 1.30 & 0 & 0 & & \\
\hline Q & $0301-0035$ & & 6 & 3.223 & 18.53 & -27.2 & & 0 & 0 & & \\
\hline$Q$ & $0301-0035$ & * & 3 & 3.205 & 18.50 & -27.2 & 0.67 & 0 & 0 & & \\
\hline Q & $0302-0019$ & & 1 & 3.286 & 18.37 & -27.4 & 1.17 & 0 & 0 & & \\
\hline $\mathrm{Q}$ & 0302-0019 & & 6 & 3.286 & 18.37 & -27.4 & & 0 & 0 & & \\
\hline Q & $0302-0019$ & * & 3 & 3.285 & 18.40 & -27.3 & 0.60 & 0 & 0 & & \\
\hline & $0302+170.5$ & * & 3 & 2.890 & 19.00 & -27.2 & 0.61 & 0 & 0 & & \\
\hline EX & $0302-223$ & * & 1 & 1.409 & 16.40 & -28.3 & 0.63 & 0 & 0 & & \\
\hline EX & $0302-223$ & & 6 & 1.409 & 16.40 & .28 .3 & & 0 & 0 & & \\
\hline$Q$ & $0304-392$ & & 1 & 1.965 & 17.60 & -27.8 & 1.14 & 0 & 0 & & \\
\hline $\mathrm{Q}$ & 0304-392 & * & 6 & 1.965 & 17.60 & .27 .8 & & 0 & 0 & & \\
\hline UM & 682 & & 1 & 2.752 & 18.30 & .27 .7 & 1.23 & 0 & 0 & & \\
\hline UM & 682 & * & 6 & 2.752 & 18.30 & -27.7 & & 0 & 0 & & \\
\hline & $0308+1902$ & * & 3 & 2.835 & 18.60 & -27.5 & 0.52 & 0 & 0 & & \\
\hline PKS & $0317-02$ & * & 3 & 2.092 & 19.50 & -26.0 & 0.65 & 0 & 0 & & \\
\hline Q & $0321-337$ & * & 1 & 1.985 & 17.81 & -27.6 & 1.18 & 0 & 0 & & \\
\hline Q & $0324-407$ & * & 6 & 3.056 & 17.60 & -28.4 & & 0 & 0 & & \\
\hline PKS & $0329-255$ & * & 1 & 2.685 & 17.51 & .28 .4 & 0.30 & 0 & 0 & & \\
\hline PKS & $0329-25.5$ & & 6 & 2.685 & 17.51 & -28.4 & & 0 & 0 & & \\
\hline Q & $0329-385$ & * & 6 & 2.423 & 16.92 & -28.7 & & 0 & 0 & & \\
\hline $\mathrm{H}$ & $0333-380$ & * & 1 & 2.201 & 18.50 & .26 .9 & 1.13 & 0 & 0 & & \\
\hline $\mathrm{H}$ & $0335-336$ & * & 1 & 2.265 & 18.50 & -26.9 & 1.12 & 0 & 0 & & \\
\hline $\mathrm{Q}$ & 0338-394 & * & 6 & 2.590 & 18.40 & -27.3 & & 0 & 0 & & \\
\hline PKS & $0347-241$ & * & 1 & 1.88 .5 & 17.50 & -27.8 & 1.24 & 0 & 0 & & \\
\hline Q & $0347-383$ & * & 1 & 3.230 & 17.30 & -28.4 & 0.84 & 0 & 0 & & \\
\hline$Q$ & 0347.383 & & 6 & 3.230 & 17.30 & -28.4 & & 0 & 0 & & \\
\hline NAB & $0348+06$ & & 5 & 2.060 & 17.60 & -27.9 & 0.87 & 0 & 0 & & \\
\hline NAB & $0348+06$ & & 6 & 2.060 & 17.60 & -27.9 & & 0 & 0 & & \\
\hline $\mathrm{NAB}$ & $0348+06$ & * & 3 & 2.058 & 17.60 & -27.9 & 0.62 & 0 & 0 & & \\
\hline UM & 684 & * & 6 & 2.819 & 18.00 & -28.1 & & 0 & 0 & & \\
\hline $\mathrm{Q}$ & 0353-383 & & 1 & 1.959 & 17.50 & -27.9 & 1.57 & 0 & 0 & & \\
\hline $\mathrm{Q}$ & 0353-383 & * & 6 & 1.959 & 17.50 & -27.9 & & 0 & 0 & & \\
\hline PKS & $0355-483$ & * & 6 & 1.016 & 16.38 & -27.5 & & 0 & 0 & & \\
\hline DW & $0400+258$ & * & 3 & 2.109 & 18.00 & -27.5 & 0.54 & 0 & 1 & 3.2 & 0.7 \\
\hline PKS & $0402-362$ & * & 6 & 1.417 & 17.17 & -27.5 & & 0 & 0 & & \\
\hline
\end{tabular}


TABLE 3. (continued)

\begin{tabular}{|c|c|c|c|c|c|c|c|c|c|c|c|}
\hline Identi & fication & $\mathrm{F}$ & Type & 2 & $\mathrm{~m}$ & M & FWHM & $P Q$ & $\mathrm{OQ}$ & $\theta_{D Q}$ & $\mathrm{MQ}$ \\
\hline SBS & $1055+.58-4$ & * & 6 & 2.239 & 18.00 & -27.4 & & 0 & 0 & & \\
\hline$Q$ & $1101-264$ & * & 2 & 2.148 & 16.02 & -29.4 & 1.02 & 0 & 0 & & \\
\hline $\mathrm{PG}$ & $1115+080$ & * & 2 & 1.724 & 16.22 & .28 .9 & 1.38 & 1 & 1 & 0.5 & 0.1 \\
\hline SBS & $1116+603$ & ${ }^{*}$ & 6 & 2.628 & 16.50 & -29.3 & & 0 & 0 & & \\
\hline SBS & $1117+535$ & ${ }^{*}$ & 6 & 1.921 & 18.00 & -27.3 & & 0 & 0 & & \\
\hline UM & 425 & $*$ & 2 & 1.465 & 16.50 & -28.3 & 1.23 & 0 & 1 & 0.0 & 0.0 \\
\hline PKS & $1123+26$ & ${ }^{*}$ & 4 & 2.341 & 18.50 & -27.3 & 0.86 & 0 & 0 & & \\
\hline Plis & $1127-1.1$ & * & 1 & $1.18 i$ & 16.90 & -27.4 & 0.89 & 0 & 0 & & \\
\hline PKS & $1127-1.1$ & & 6 & 1.187 & 16.90 & $-2 \pi .4$ & & 0 & 0 & & \\
\hline & $1136+121.1$ & * & 2 & 2.89 .4 & 17.60 & -28.6 & $1.3 \bar{\tau}$ & 0 & 0 & & \\
\hline & $1136+121+1$ & & 6 & 2.894 & 17.60 & .28 .6 & & 0 & 0 & & \\
\hline & $1136+1214$ & & 4 & 2.894 & 17.60 & -28.6 & 0.79 & 0 & 0 & & \\
\hline us & 2778 & $*$ & 6 & 1.578 & 16.90 & -28.0 & & 0 & 0 & & \\
\hline SBS & $1138+584$ & $*$ & 6 & 1.699 & 18.00 & -27.1 & & 0 & 0 & & \\
\hline PG & $1138+040$ & * & 2 & 1.877 & 17.19 & -28.1 & 1.12 & 0 & 0 & & \\
\hline PG & $1138+0.40$ & & 5 & 1.877 & 17.19 & -28.1 & 0.78 & 0 & 0 & & \\
\hline is & 2813 & $*$ & 6 & 1.691 & 17.60 & -27.5 & & 0 & 0 & & \\
\hline Plis & $11+8-00$ & & 1 & $1.98 \%$ & 17.60 & -27.8 & 1.43 & 0 & 0 & & \\
\hline Plis & $1118-00$ & : & 6 & 1.982 & 17.60 & -27.8 & & 0 & 0 & & \\
\hline B.2 & $1118+38$ & ${ }^{*}$ & 6 & 1.30 .3 & 17.04 & -27.5 & & 0 & 0 & & \\
\hline POX & $.5 \mathrm{~B}$ & 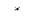 & 2 & $2.20 \bar{i}$ & 17.00 & .28 .4 & 1.61 & 0 & 0 & & \\
\hline Plis & $11.57+01 .+$ & ${ }^{*}$ & 6 & 1.986 & 17.74 & -27.6 & & 0 & 0 & & \\
\hline POX & +2 & $*$ & 1 & 2.453 & 17.01 & -28.5 & 0.99 & 0 & 0 & & \\
\hline Q & $1159+123$ & * & 2 & 3.502 & 17.50 & .28 .4 & 1.14 & 0 & 1 & 0.5 & 0.2 \\
\hline Q & $1159+123$ & & 6 & 3.502 & 17.50 & .28 .4 & & 0 & 0 & & \\
\hline POX & 61 & * & 6 & 2.455 & 17.80 & -27.7 & & 0 & 0 & & \\
\hline$P G$ & $1206+459$ & $*$ & 6 & 1.158 & 16.07 & -28.1 & & 0 & 0 & & \\
\hline & $1206+1155$ & * & (i & 3.106 & 17.90 & -28.0 & & 0 & 0 & & \\
\hline & $12076+3999$ & * & 1 & 2.400 & 17.50 & .27 .8 & 1.17 & 0 & 0 & & \\
\hline & $1208+1011$ & " & 2 & $3.80: 3$ & 17.50 & -28.7 & 1.38 & 0 & 1 & 0.4 & 1.4 \\
\hline & $1208+1011$ & & 6 & 3.802 & 17.50 & -28.7 & & 0 & 1 & 0.4 & 1.3 \\
\hline$Q$ & $1213+0922$ & * & 1 & 2.719 & $17: 20$ & -28.7 & 0.94 & 0 & 0 & & \\
\hline$Q$ & $1213+0922$ & & 6 & 2.719 & 17.20 & -28.7 & & 0 & 0 & & \\
\hline UM & 485 & * & 1 & 2.690 & 17.00 & -28.9 & 1.49 & 0 & 0 & & \\
\hline$B 2$ & $1215+33$ & & 5 & 2.606 & 17.50 & .28 .3 & 1.11 & 0 & 0 & & \\
\hline B2 & $121.5+33$ & $*$ & 6 & 2.606 & 17.50 & 28.3 & & 0 & 0 & & \\
\hline $\mathrm{MC}$ & $1215+11: 3$ & * & 6 & 1.396 & 16.86 & -27.8 & & 0 & 0 & & \\
\hline SBS & $1221+54$ & . & 6 & 2.106 & 18.00 & -27.5 & & 0 & 0 & & \\
\hline & $1222+023$ & "' & 1 & 2.050 & 17.00 & -28.1 & 0.99 & 0 & 0 & & \\
\hline $70 \times$ & $15: 30$ & $=$ & i & 2.051 & 16.60 & -28.8 & 0.87 & 0 & 0 & & \\
\hline B.2 & $1225+31$ & * & i) & 2.219 & 15.87 & -29.5 & 1.14 & 0 & 0 & & \\
\hline & $1228 . \bar{i}+0 \bar{i} . \bar{i}$ & * & 6 & 2.391 & 17.59 & $\cdot 28.0$ & & 0 & 0 & & \\
\hline$P G_{i}$ & $12: 11+176$ & * & 1 & 1.27 .3 & 16.30 & -28.2 & 0.95 & 0 & 1 & 3.1 & 6.5 \\
\hline PG & $1241+176$ & & 6 & 1.273 & 16.30 & -28.2 & & 0 & 0 & & \\
\hline & $1244.9+34.7$ & * & 6 & 2.480 & 18.00 & -27.6 & & 0 & 0 & & \\
\hline BSO & 1 & * & 6 & 1.241 & 16.98 & -27.4 & & 0 & 0 & & \\
\hline $\mathrm{Q}$ & $1246-0.57$ & $=$ & 1 & 2.212 & 16.73 & -28.7 & 1.25 & 0 & 0 & & \\
\hline PG & $124 \bar{\tau}+268$ & * & 5 & 2.041 & 15.90 & -29.5 & 0.76 & 0 & 0 & & \\
\hline PG & $1247+268$ & & 6 & 2.041 & 15.90 & -29.5 & & 0 & 0 & & \\
\hline PG & $1254+047$ & * & 6 & 1.024 & 16.14 & -27.7 & & 0 & 0 & & \\
\hline B & 201 & ${ }^{x}$ & 6 & 1.375 & 16.79 & -27.8 & & 0 & 0 & & \\
\hline w & 61972 & ${ }^{x}$ & 6 & 1.922 & 17.75 & -27.6 & & 0 & 0 & & \\
\hline BSO & 6 & * & 6 & 1.956 & 17.87 & -27.5 & & 0 & 0 & & \\
\hline w & 22722 & * & 6 & 1.759 & 17.85 & -27.3 & & 0 & 0 & & \\
\hline W & 21541 & $\approx$ & 6 & $2.04 \pi$ & 17.72 & -27.7 & & 0 & 0 & & \\
\hline POX & 123 & * & 6 & 2.289 & 18.00 & 27.7 & & 0 & 0 & & \\
\hline $4 \mathrm{C}$ & 18.36 & * & 5 & 1.689 & 18.50 & .26 .6 & 0.89 & 0 & 0 & & \\
\hline$Q$ & $1309-0.56$ & $*$ & 2 & 2.185 & 17.44 & -28.0 & 1.05 & 0 & 0 & & \\
\hline$Q$ & $1309-056$ & & 6 & 2.18 .5 & 17.44 & -28.0 & & 0 & 0 & & \\
\hline Plis & $1311 \cdot 270$ & * & 1 & 2.195 & 17.43 & -28.0 & 0.75 & 0 & 0 & & \\
\hline Plis & $1311-270$ & & 6 & 2.19 .5 & 17.43 & .28 .0 & & 0 & 0 & & \\
\hline BSO & 11 & * & 3 & 2.084 & 18.40 & -27.1 & 0.68 & 0 & 0 & & \\
\hline lM & 557 & $*$ & 1 & 2.687 & 18.60 & 27.3 & 1.09 & 0 & 0 & & \\
\hline SBS & $1315+605$ & * & 6 & 1.981 & 18.00 & -27.4 & & 0 & 0 & & \\
\hline $\mathrm{PC}$ & $1315++722$ & * & 3 & 2.592 & 18.00 & -27.7 & 0.60 & 0 & 0 & & \\
\hline TON & 153 & * & 6 & 1.022 & 15.98 & -27.9 & & 0 & 0 & & \\
\hline Q & $1317-0.507$ & * & 1 & 3.700 & 18.10 & -28.0 & 1.00 & 0 & 0 & & \\
\hline$Q$ & $1318-113$ & * & 6 & 2.308 & 17.68 & -27.4 & & 0 & 0 & & \\
\hline PKS & $1318+111$ & * & 1 & 2.172 & 19.13 & -26.3 & 1.30 & 0 & 1 & 1.5 & 3.0 \\
\hline TON & 155 & & 6 & 1.703 & 17.27 & -27.8 & & 0 & 0 & & \\
\hline TON & 15.5 & $*$ & 5 & 1.703 & 17.27 & .27 .8 & 0.87 & 0 & 0 & & \\
\hline POX & 188 & $*$ & 2 & 2.360 & 17.00 & -28.8 & 1.00 & 0 & 0 & & \\
\hline$Q$ & $1323-0248$ & * & 1 & 2.121 & 17.40 & 28.1 & 0.90 & 0 & 0 & & \\
\hline Plis & $1327-206$ & * & 6 & 1.165 & 17.04 & -27.2 & & 0 & 0 & & \\
\hline$P G_{i}$ & $1329+412$ & * & 4 & 1.935 & 17.18 & 28.2 & 1.10 & 0 & 0 & & \\
\hline $\mathrm{Q}$ & $1330+0108$ & * & 2 & 3.510 & 18.20 & -27.8 & 1.38 & 0 & 0 & & \\
\hline$M C$ & $1331+170$ & * & 2 & 2.084 & 16.71 & 28.8 & 1.29 & 0 & 0 & & \\
\hline $\mathrm{MC}$ & $1331+170$ & & 5 & 2.084 & 16.71 & -28.8 & 0.91 & 0 & 0 & & \\
\hline UM & 590 & * & 2 & 2.790 & 17.00 & -29.0 & 0.88 & 0 & 1 & 0.2 & 0.5 \\
\hline & $1337+1121$ & * & 6 & 2.919 & 18.20 & .28 .0 & & 0 & 0 & & \\
\hline Q & $1338-018$ & * & 1 & 2.079 & 17.40 & .28 .1 & 1.00 & 0 & 0 & & \\
\hline & $1340+09.59$ & * & 2 & 2.942 & 18.50 & .27 .7 & 1.26 & 0 & 0 & & \\
\hline $4 C$ & 58.27 & * & 6 & 2.039 & 17.50 & -27.9 & & 0 & 0 & & \\
\hline$Q$ & $1346-0.36$ & & 2 & 2.345 & $17.2 \bar{\tau}$ & -28.5 & 1.94 & 0 & 0 & & \\
\hline Q & $13: 16-036$ & * & 6 & 2.315 & 17.27 & -28.5 & & 0 & 0 & & \\
\hline & $134 \pi+1116$ & * & 6 & 2.697 & 18.50 & -27.4 & & 0 & 0 & & \\
\hline
\end{tabular}


TABLE 3. (continued)

\begin{tabular}{|c|c|c|c|c|c|c|c|c|c|c|c|c|c|c|c|c|c|c|c|c|c|c|c|}
\hline \multicolumn{2}{|c|}{ Identification } & $\mathrm{F}$ & Type & 2 & $\mathrm{~m}$ & M & FWHM & $\mathrm{PQ}$ & $\mathrm{OQ}$ & $\theta_{D Q}$ & $\mathrm{MQ}$ & Identi & fication & $\mathrm{F}$ & Type & $z$ & $\mathrm{~m}$ & $M$ & FWHM & PQ & $O Q$ & $\theta_{D Q}$ & $\mathrm{MQ}$ \\
\hline Q & $20 \cdot 49-353$ & $x^{x}$ & 1 . & 3.040 & 18.45 & -27.6 & 1.10 & 0 & 0 & & & $Q$ & $22409-3702$ & * & 1 & 1.820 & 18.00 & -27.2 & 0.69 & 0 & 0 & & \\
\hline Q & $2050-359$ & * & 1 & 3.490 & 18.31 & 27.6 & 1.30 & 0 & 0 & & & UM & 658 & ${ }^{*}$ & 6 & 2.850 & 18.10 & 28.0 & & 0 & 0 & & \\
\hline Q & $20.51-37.3$ & * & 1 & 2.590 & 17.22 & 28.0 & 1.20 & 0 & 0 & & & PKS & $2245-328$ & $*$ & 1 & 2.268 & 18.60 & -26.8 & 1.10 & 0 & 0 & & \\
\hline Q & 20.51 .355 & $*$ & 1 & 3.310 & 18.30 & -27.1 & 1.10 & 0 & 0 & & & PKS & $2246-309$ & * & 1 & $1.30 \mathrm{~T}$ & 17.00 & -27.5 & 0.73 & 0 & 0 & & \\
\hline Q & $2055-1.10$ & ${ }^{*}$ & 6 & 2.063 & 17.90 & $-2 \pi .6$ & & 0 & 0 & & & $Q$ & $2246-389$ & * & 2 & 2.120 & 17.90 & $-27.0^{\circ}$ & 1.39 & 0 & 0 & & \\
\hline & $21111.1+0629$ & x & 3 & 2.330 & 19.80 & -25.9 & 0.66 & 0 & 0 & & & $Q$ & $2246-389$ & & 6 & 2.120 & 17.90 & -27.6 & & 0 & 0 & & \\
\hline Q & $2112-10 \overline{7}$ & * & 1 & 2.513 & 18.00 & $-2 \pi .7$ & 1.10 & 0 & 0 & & & $4 \mathrm{C}$ & 19.74 & * & 4 & 1.806 & 18.70 & -26.5 & 0.80 & 0 & 0 & & \\
\hline Plis & 2115.305 & * & 1 & 0.980 & 16.47 & -27.3 & 1.20 & 0 & 1 & 3.0 & 2.0 & Plis & $2251+24$ & & 5 & 2.328 & 17.80 & -28.0 & 0.61 & 0 & 0 & & \\
\hline Q & $2116-358$ & & 2 & 2.341 & 17.35 & 28.4 & 1.50 & 0 & 0 & & & PKS & $2251+24$ & & 6 & 2.328 & 17.80 & -28.0 & & 0 & 0 & & \\
\hline Q & $211(i-358$ & * & 6 & 2.341 & 17.35 & 28.4 & & 0 & 0 & & & PKS & $2251+24$ & * & 3 & 2.328 & 17.80 & -28.0 & 0.48 & 0 & 0 & & \\
\hline & $21189+168$ & $\times$ & 3 & 2.300 & 19.50 & -26.2 & $0.5 !$ & 0 & 1 & 4.6 & 2.8 & PKS & $2254+024$ & & 2 & 2.090 & 17.00 & .28 .5 & 1.53 & 0 & 0 & & \\
\hline $3 C^{\circ}$ & 132.0 & × & 3 & $1.80 \%$ & 18.00 & -27.2 & 0.71 & 0 & 0 & & & PKS & $2254+024$ & & 5 & 2.089 & 17.00 & -28.5 & 0.62 & 0 & 0 & & \\
\hline plis & $2121+0.53$ & & 1 & 1.878 & 17.50 & 27.8 & 0.81 & 0 & 0 & & & Plis & $2254+024$ & & 6 & 2.090 & 17.00 & -28.5 & & 0 & 0 & & \\
\hline Plis & $2121+0.53$ & & 6 & 1.878 & 17.50 & -27.8 & & 0 & 0 & & & Plis & $2254+024$ & * & 3 & 2.090 & 17.00 & .28 .5 & 0.58 & 0 & 0 & & \\
\hline PISS & $2|2|+053$ & & 1 & 1.878 & 17.50 & -27.8 & 1.05 & 0 & 0 & & & PISS & $2256+017$ & & 4 & 2.663 & 19.00 & -26.8 & 0.63 & 0 & 0 & & \\
\hline Plis & $2121+0.53$ & x & 3 & 1.878 & 17.50 & -27.8 & 0.54 & 0 & 0 & & & PKS & $2256+01 \bar{\imath}$ & * & 3 & 2.663 & 19.00 & -26.8 & 0.55 & 0 & 0 & & \\
\hline Q & $2123-108$ & $\approx$ & 1 & 2.290 & 18.50 & -27.2 & 0.80 & 0 & 0 & & & $\mathrm{Q}$ & $2258-391$ & * & 6 & 2.050 & 18.00 & .27 .4 & & 0 & 0 & & \\
\hline$Q$ & $2125-1335$ & - & 1 & 2.948 & 18.70 & -27.5 & 0.90 & 0 & 0 & & & Q & $2300-352$ & & 2 & 2.840 & 17.90 & $\cdot 28.2$ & 1.64 & 0 & 0 & & \\
\hline PISS & $2126-1.5$ & * & 2 & 3.275 & 17.30 & -28.4 & 1.38 & 0 & 0 & & & $Q$ & $2300-352$ & * & 6 & 2.840 & 17.90 & -28.2 & & 0 & 0 & & \\
\hline Plis & $2126-15$ & & 6 & 3.275 & 17.30 & -28.4 & & 0 & 0 & & & $\mathrm{PG}$ & $2302+029$ & * & 1 & 1.044 & 16.10 & -27.8 & $0 . \bar{\imath} 1$ & 0 & 0 & & \\
\hline $\mathrm{HA}$ & 2 & * & 1 & 2.093 & 17.5 .5 & .27 .9 & 1.30 & 0 & 0 & & & PG & $2302+029$ & & 6 & 1.044 & 16.10 & -27.8 & & 0 & 0 & & \\
\hline Plis & $2134+001$ & * & 1 & 1.936 & 16.79 & 28.5 & 0.68 & 0 & 0 & & & TEX & $2303+183$ & $*$ & 4 & 1.557 & 18.00 & -26.9 & 0.63 & 0 & 0 & & \\
\hline PKS & $213.1+001$ & & 5 & 1.936 & 16.79 & .28 .5 & 0.68 & 0 & 0 & & & $Q$ & $2304-423$ & * & 6 & 2.630 & 17.60 & -28.2 & & 0 & 0 & & \\
\hline PKS & $213-1+001$ & & (6) & 1.936 & 16.79 & -28.5 & & 0 & 0 & & & PKS & $2319+07$ & * & 4 & 2.090 & 18.50 & -27.0 & 0.76 & 0 & 0 & & \\
\hline Plis & $213.4+00-4$ & & 3 & 1.936 & 16.80 & -28.5 & $0 . i t$ & 0 & 1 & 0.0 & 0.0 & UM & 164 & & 6 & 1.900 & 17.00 & -28.3 & & 0 & 0 & & \\
\hline & $213+2-149$ & * & + & 2.200 & 18.30 & -27.1 & 0.69 & 0 & 0 & & & UM & 164 & * & 3 & 1.900 & 17.00 & -28.3 & 0.63 & 0 & 0 & & \\
\hline PKS & $21.36+1.41$ & & 6 & $2.42 \bar{\tau}$ & 18.50 & $2 \pi .1$ & & 0 & 0 & & & & $23333+019 \mathrm{~B}$ & & 4 & 1.871 & 18.00 & -27.3 & 0.87 & 0 & 0 & & \\
\hline PKS & $2136+1.41$ & . & 3 & 2.427 & 18.50 & -27.1 & 0.48 & 0 & 0 & & & & $23333+019 \mathrm{~B}$ & $=$ & 3 & 1.871 & 18.00 & -27.3 & 0.75 & 0 & 0 & & \\
\hline & $21431+0.40$ & * & 3 & 2.000 & 19.20 & -26.2 & 0.66 & 0 & 0 & & & Plis & $2335-181$ & $=$ & 6 & 1.441 & 17.34 & -27.4 & & 0 & 0 & & \\
\hline Plis & $21+4-362$ & . & 6 & 2.081 & 17.80 & $-27 . \bar{i}$ & & 0 & 0 & & & PKS & $23335-181$ & & 1 & 1.441 & 17.34 & .27 .4 & 1.10 & 0 & 0 & & \\
\hline PKS & $2119-306$ & * & 6 & 2.345 & 18.00 & $27 . \overline{7}$ & & 0 & 0 & & & PKS & $2338+042$ & * & 3 & 2.592 & 19.50 & -26.2 & 0.94 & 0 & 0 & & \\
\hline PKS & $21.50+0.5$ & & 6 & 1.979 & 17.77 & .27 .6 & & 0 & 0 & & & $\mathrm{BG}$ & CFH 26 & * & 3 & 2.140 & 19.70 & -25.8 & 0.58 & 0 & 0 & & \\
\hline PKS & $21.50+0.5$ & & 1 & 1.979 & 17.77 & -27.6 & $0.6 \bar{i}$ & 0 & 0 & & & $B G$ & CFH 27 & * & 3 & 2.340 & 18.70 & -27.0 & 0.61 & 0 & 0 & & \\
\hline PKS & $21.50+0.5$ & * & 3 & 1.979 & 17.80 & 27.6 & 0.53 & 0 & 0 & & & UM & 660 & * & 6 & 2.822 & 18.10 & -28.0 & & 0 & 0 & & \\
\hline PKS & $2153-20.1$ & * & 1 & 1.309 & 17.01 & -27.5 & 0.65 & 0 & 0 & & & UM & 175 & & 6 & 1.960 & 18.60 & -26.8 & & 0 & 0 & & \\
\hline Plis & $2153-20.1$ & & 6 & 1.309 & 17.01 & -27.5 & & 0 & 0 & & & UM & 175 & * & 3 & 1.960 & 18.00 & -27.4 & 0.58 & 0 & 0 & & \\
\hline B2 & $2156+29$ & & 6 & 1.753 & 17.50 & -27.6 & & 0 & 0 & & & $B G$ & Clll 35 & $=$ & 3 & 2.700 & 18.90 & -27.0 & 0.48 & 0 & 0 & & \\
\hline B2 & $2156+29$ & * & 3 & 1.7 .53 & 17.50 & -27.6 & 0.58 & 0 & 0 & & & $B \mathrm{~B}^{-}$ & ( FH H H & $\times$ & 3 & 2.100 & 19.80 & 25.7 & 0.48 & 0 & 0 & & \\
\hline & $21.58-2140$ & ** & 6 & 2.079 & 18.10 & -27.4 & & 0 & 0 & & & UM & 180 & * & 4 & 1.960 & 18.00 & -27.4 & 0.87 & 0 & 0 & & \\
\hline$M C:$ & $21.58+101$ & * & 4 & 1.727 & 18.70 & -26.4 & 0.77 & 0 & 0 & & & PKS & $2345+061$ & & 6 & 1.5 .16 & 17.50 & -27.4 & & 0 & 0 & & \\
\hline $\mathrm{PB}$ & 5062 & ** & 2 & 1.710 & 17.50 & .27 .7 & 1.13 & 0 & 0 & & & PliS & $2345+061$ & * & 4 & 1,538 & 17.50 & -27.4 & $0.6 \bar{T}$ & 0 & 0 & & \\
\hline & $2 \cdot 203+29$ & . & 3 & 4.406 & 2.00 & -24.5 & $0.5 i$ & 0 & 0 & & & UM & 184 & & 6 & 3.00 .5 & 19.50 & -26.6 & & 0 & 0 & & \\
\hline Plis & $220-1-573$ & & 2 & 2.72 .5 & 17.36 & -28.6 & 1.82 & 0 & 0 & & & UM & 184 & * & 4 & 3.005 & 19.50 & 26.6 & 0.62 & 0 & 0 & & \\
\hline PKS & $2204-573$ & * & 6 & 2.72 .5 & 17.36 & -28.6 & & 0 & 0 & & & $Q$ & $2348-4025$ & * & 1 & 3.310 & 18.60 & .27 .1 & 0.99 & 0 & 0 & & \\
\hline $\mathrm{Q}$ & $2 \cdot 24-408$ & & 2 & 3.170 & $17.5 \bar{T}$ & -28.2 & 2.09 & 0 & 0 & & & $Q$ & $2348-4025$ & & 6 & 3.310 & 18.60 & .27 .1 & & 0 & 0 & & \\
\hline Q & $220+1-408$ & ${ }^{*}$ & 6 & 3.170 & 17.57 & 28.2 & & 0 & 0 & & & WEE & 180 & $*$ & 3 & 2.040 & 19.80 & .25 .6 & 0.67 & 0 & 0 & & \\
\hline & $206-199 \mathrm{~N}$ & ${ }^{*}$ & 1 & 2.5 .59 & 17.33 & .28 .4 & 0.70 & 0 & 0 & & & $\mathrm{Q}$ & $2: 3-49-012$ & x & 1 & 3.120 & 16.50 & .29 .3 & 1.00 & 0 & 0 & & \\
\hline & $2209-18 \pi 1$ & & 1 & 2.092 & 17.80 & -27.7 & 1.36 & 0 & 0 & & & PKS & $2351-15.1$ & * & 2 & 2.666 .5 & 17.00 & -28.9 & 1.20 & 0 & 0 & & \\
\hline & $2209-187 \mathrm{U}$ & * & 6 & 2.092 & 17.80 & -27.7 & & 0 & 0 & & & PKS & $2351-151$ & & 6 & 2.666 .5 & 17.00 & 28.9 & & 0 & 0 & & \\
\hline PKS & $2212-299$ & * & 1 & 2.703 & 17.44 & -28.5 & 0.69 & 0 & 0 & & & PKS & $23552-455$ & * & 2 & 1.868 & 17.50 & -27.8 & 1.01 & 0 & 0 & & \\
\hline PLiS & $2212-299$ & & 6 & 2.703 & 17.44 & -28.5 & & 0 & 0 & & & PKS & $2352-455$ & & 6 & 1.868 & 17.50 & -27.8 & & 0 & 0 & & \\
\hline $\mathrm{Q}$ & $2219-394$ & & 1 & 2.022 & 17.74 & -27.7 & 1.26 & 0 & 0 & & & Plis & $2.353+15.1$ & & 1 & 1.801 & 18.00 & -27.2 & 0.69 & 0 & 0 & & \\
\hline Q & $2219-394$ & * & 6 & 2.022 & 17.74 & -27.7 & & 0 & 0 & & & PKS & $2353+154$ & 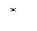 & 3 & 1.801 & 18.00 & -27.2 & 0.63 & 0 & 0 & & \\
\hline PKS & $2222+05$ & * & 3 & 2.324 & 18.50 & -27.2 & 0.75 & 0 & 0 & & & PKS & $2354+14$ & & 4 & 1.810 & 18.18 & -27.0 & 0.62 & 0 & 0 & & \\
\hline$Q$ & $22 \cdot 2-396$ & $*$ & 1 & 2.180 & 17.90 & .27 .5 & 0.65 & 0 & 0 & & & PKS & $2354+14$ & * & 3 & 1.810 & 18.20 & -27.0 & 0.42 & 0 & 0 & & \\
\hline Q & $2222-396$ & & 6 & 2.180 & 17.90 & -27.5 & & 0 & 0 & & & PKS & $23.54+14$ & & 1 & 1.810 & 18.18 & -27.0 & 0.90 & 0 & 0 & & \\
\hline PKS & $2223+21$ & & 6 & 1.959 & 18.00 & -27.4 & & 0 & 0 & & & $Q$ & $235.5-025$ & × & 1 & 2.0 .50 & 17.50 & -27.9 & 1.00 & 0 & 0 & & \\
\hline Plis & $2223+21$ & * & 3 & 1.959 & 18.00 & -27.4 & 1.00 & 0 & 0 & & & $Q$ & $2355-389$ & * & 6 & 2.850 & 18.40 & -27.7 & & 0 & 0 & & \\
\hline $\mathrm{Q}$ & $2225-404$ & * & 1 & 2.020 & 18.28 & -27.1 & 0.70 & 0 & 0 & & & PKS & $2355-106$ & * & 4 & 1.622 & 18.50 & -26.5 & 1.13 & 0 & 0 & & \\
\hline PHL & 5200 & * & 2 & 1.981 & 17.70 & -27.7 & 0.64 & 0 & 0 & & & $\mathrm{Q}$ & $2355-019$ & * & 1 & 1.640 & 16.50 & -28.5 & 1.00 & 0 & 1 & 1.9 & 3.0 \\
\hline PHL & 5200 & & 6 & 1.981 & 17.70 & -27.7 & & 0 & 0 & & & $Q$ & $2355+019$ & * & 1 & 2.790 & 17.50 & -28.5 & 1.00 & 0 & 0 & & \\
\hline PHL & 5200 & & 4 & 1.981 & 17.70 & -27.7 & 0.65 & 0 & 0 & & & UM & 193 & ${ }^{\star}$ & 1 & 2.070 & 18.40 & -27.1 & 1.21 & 0 & 0 & & \\
\hline PHL & 5200 & & 3 & 1.981 & 17.70 & -27.7 & 0.65 & 0 & 0 & & & UM & 193 & & + & 2.070 & 18.40 & .27 .1 & 0.81 & 0 & 0 & & \\
\hline PKS & $2227-08$ & * & 1 & 1.562 & 17.50 & -27.4 & 0.65 & 0 & 0 & & & $\mathrm{Q}$ & $2357-3.48$ & * & 1 & 2.070 & 17.78 & -27.7 & 1.00 & 0 & 0 & & \\
\hline PKS & $2227-08$ & & 5 & 1.562 & 17.50 & -27.4 & 0.72 & 0 & 0 & & & $Q$ & $2357-345$ & & 6 & 2.070 & 17.78 & -27.7 & & 0 & 0 & & \\
\hline $\mathrm{Q}$ & $2228-399$ & * & 1 & 2.200 & 18.30 & -27.1 & 0.81 & 0 & 0 & & & PLS & $2357-326$ & $\times$ & 6 & 1.27 .5 & 17.00 & -27.5 & & 0 & 0 & & \\
\hline & $2233.9+1318$ & * & 3 & 3.280 & 18.20 & -27.5 & 0.46 & 0 & 0 & & & PKS & $2358-161$ & ${ }^{x}$ & 6 & 2.044 & 18.00 & -27.4 & & 0 & 0 & & \\
\hline & $2237.9+0040$ & . & 3 & 2.200 & 18.40 & -27.0 & 0.72 & 0 & 0 & & & & $2359+0653$ & * & 6 & 3.234 & 18.80 & .26 .9 & & 0 & 0 & & \\
\hline UM & 657 & * & 1 & 1.360 & 17.20 & -27.4 & 0.83 & 0 & 0 & & & UM & 196 & & 4 & 2.817 & 18.70 & -27.4 & 0.67 & 0 & 0 & & \\
\hline UM & 657 & & 6 & 1.360 & 17.20 & -27.4 & & 0 & 0 & & & UM & 196 & & 6 & 2.817 & 18.00 & -28.1 & & 0 & 0 & & \\
\hline & $2239.5+0073$ & $\times$ & 3 & 2.400 & 19.80 & -26.1 & 0.45 & 0 & 0 & & & UM & 196 & * & 3 & 2.820 & 18.00 & -28.1 & 0.71 & 0 & 0 & & \\
\hline Q & $2240-419$ & * & 6 & 2.080 & 18.00 & -27.5 & & 0 & 0 & & & & & & & & & & & & & & \\
\hline
\end{tabular}

Notes:

Col. 2: An asterisk $\left({ }^{*}\right)$ in this column indicates which of the listed observation(s) for a same HLQ turned out to be the best one for calculating, in section 3, the effectiveness parameter F" of galaxies to produce multiply lensed quasar images

Col. 3: Type as defined in Table 2

Col. 4: Recdshift $z$ of the $11 \mathrm{LO}$

(ol. 5: $m$ is the apparent magnitude of the HLOs as listed under the column $V$ in the catalogue of Veron-('chy and Veron (1991). Unfortunately, these magnitudes are not always "visual" ones; they are generally quite inaccurate and inlomogeneous.

Col. 6: M stands for absolute visual magnitudes, calculated from the apparent magnitudes $\mathrm{m}$ (see ( Col. j) and assuming $H_{v}=50 \mathrm{~km} / \mathrm{sec} / \mathrm{Mpc}$ and $q_{0}=0.5$. Note that $\mathrm{k}$-corrections were applied witl $\alpha=-0.7$ and a magnitude correction for the presence of broad emission lines (see Véron-('etty and Véron 1991).

Col. 7 : FWHM corresponds to the full width at half maximum, in arcsec., of stellar images recorded on the ('CD) frames.

Cols. 8. 9: Indications on whether the HLQ consists of a confirmed GL $(P Q=1)$ or of just a possible candidate $(O Q=1$. but $P Q=0$. for such candidates)

Cols. $10 \mathrm{~d}: 1 /:$ If $P Q=1$ or $O Q=1$, we list here the distance $\theta_{D Q}$ (in arcsec) and the magnitude difference $.1 Q$ between the identified secondary point source image and the primary one. Null values in these columus indicate that the relevant quantities could not be determined satisfactorily: (cf. the position of a faint residual obtained from a PSF subtraction) 
(iv) when deriving the expression of $\tau$, TOG assume that there is no evolution of the lens properties with cosmic time [i.e., $n(z) \propto n(0)(1+z)^{3}$ ]. Mao (1991) has shown that a simple redshift cutoff model for the lensing galaxies may significantly reduce the lensing probability and also explain the large mean separation of images for known lenses such as $0957+561,2345+007$, etc. Mao also warns that such an effect could mask the contribution of a nonnegligible cosmological constant $\Lambda$ in producing the apparent observed number of lenses,

(v) the above derivation of $\tau$ is only valid for the case of an isolated lens. Jaroszynski (1991) has shown numerically that the grouping of galaxies and possible correlations between galaxies and background matter density may increase the probability of high amplifications. Other possible intervening density inhomogeneities (cf. granularity in the galaxies, cluster substructure at kpc scales, etc.) could also be of some importance but were not taken into account because of lack of better knowledge;

(vi) probably of great importance is the inadequacy of the SIS model to account for the observed GL configurations which consist of more than two images, and thus it cannot be expected to give a very precise estimate of $\tau$, neither of the magnification bias. Using an elliptical lens model with realistic physical parameters (core radius, ellipticity, etc.) and by means of extensive Monte-Carlo simulations, Kochanek (1991b) and Wallington \& Narayan (1992) have recently proposed more accurate estimates of $\tau$. It would certainly be very appropriate to apply in the future their formalism to the observational results compiled in the present paper;

(vii) Finally, it should be noted that the sensitivity of $\tau$ upon the redshift-distance formulation and statistics formalism has been investigated by FFKT. Based upon numerical simulations (provided that there are no sources at very large redshifts, i.e., $z_{Q} \leqslant 3$ ), they conclude that the formulation dependence is small in the final prediction of lenses. Note also that $\tau$ is independent of $H_{0}$ and also on the possible extra lensing brought by galaxy clusters since we did not consider GL candidates with image separations greater than $\simeq 3^{\prime \prime}$.

On account of part of the complications listed above, Kochanek (1991b) has estimated that the error affecting the optical depth $\tau$ for lensing should be of the order of at least a factor 4 .

We therefore conclude that our results on the expected number $N^{\text {exp }}$ of GLs (cf. Table 4) agree sufficiently well with current expectations (cf. FT) and that it is not justified at the present time to compare observed to estimated numbers of multiply imaged quasars in order to infer the values of cosmological parameters such as $\Lambda, \Omega_{0}$, etc.

\subsection{Compact Lenses}

\subsubsection{The method}

Press \& Gunn (1973) have pointed out that the frequency of lensing events caused by a population of compact objects measures their contribution $\Omega_{L}$ to $\Omega_{0}, \Omega_{L}$ being the local density of lensing points in units of the critical

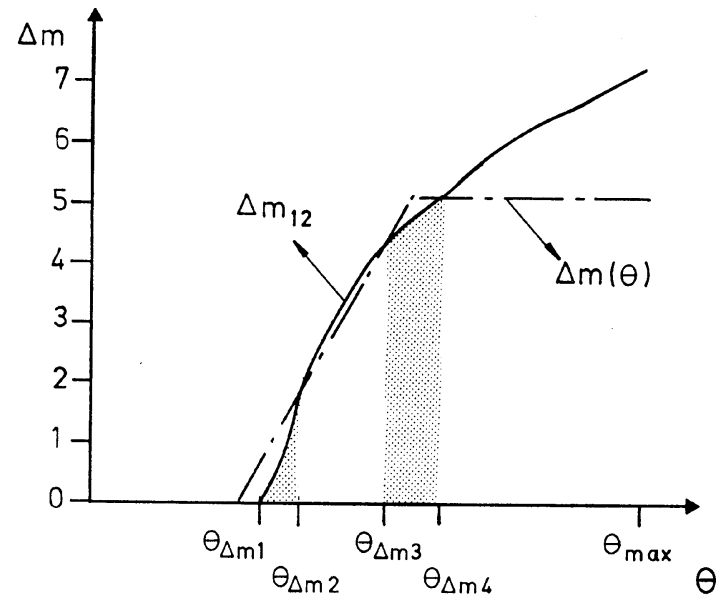

FIG. 3. One example of possible multiple intersections $\left(\theta_{\Delta m i r}\right.$ with generally $i \leqslant 4$ ) between the "angular selection function" $\Delta m(\theta)$ (cf. Fig. 2) and the predicted magnitude difference $\Delta m_{12}$ between the two lensed images of a distant quasar. Note that $\theta_{\Delta m 1}=2 \theta_{E}$, where $\theta_{E}$ represents the angular radius of the Einstein ring associated with the compact lens. $\theta_{\max }$ $\left(=3^{\prime \prime}\right)$ is the maximum angular radius of the field under consideration (see text).

density. Adopting the "detection volume" formalism developed by Nemiroff (1991a,b) and Kassiola et al. (1991) in order to derive an expression for the lensing optical depth $\tau_{Q}$ of a distant quasar by a hypothetical population of compact lenses in the Universe, with mass $\mathscr{M}_{L}$, it is easy to establish that

$$
\begin{aligned}
\tau_{Q}= & \pi \int_{0}^{D_{O Q}^{P}} n\left(D_{O L}^{P}\right)\left[\operatorname{Min}\left(b_{\Delta m 2}^{2}, b_{\max }^{2}\right)-\operatorname{Min}\left(b_{\Delta m 1}^{2}, b_{\max }^{2}\right)\right. \\
& \left.+\operatorname{Min}\left(b_{\Delta m 4}^{2}, b_{\max }^{2}\right)-\operatorname{Min}\left(b_{\Delta m 3}^{2}, b_{\max }^{2}\right)\right] d D_{O L}^{P},
\end{aligned}
$$

$\tau_{Q}(\ll 1)$ representing in fact the expected number of lenses, distributed with a cosmic density $n\left(z_{L}\right)=n_{0}\left(1+z_{L}\right)^{3}$ at a proper distance $D_{O L}^{P}$ from the observer, inside a volume such that they are capable of producing observable double images of a distant quasar $Q$. In the above expression for $\tau_{Q}$, several observational biases are implicitly taken into account. For instance, we have constrained the lensed images to be characterized by a magnitude difference smaller than $\Delta m$, which is itself a function of $\theta$ (cf. Fig. 2, see also the $\Delta m-\theta$ relations given in Table 2 ) and by a maximum angular separation $\theta_{\max }$ (set to $3^{\prime \prime}$ in our calculations). In the above equation, the quantities $b_{\Delta m i}(i=1-4)$ and $b_{\max }$ represent extreme impact parameters defined in the lens plane such that $b_{\Delta m i}=D_{O L} \theta_{\Delta m i}$ (respectively, $b_{\max }$ $\left.=D_{O L} \theta_{\max }\right)$, where $D_{O L}$ is the angular distance of the lens as measured by the observer and where the $\theta_{\Delta m i}(i=1-4$, respectively $\theta_{\max }$ ) represent possible intersecting points between the $\Delta m-\theta$ function (cf. Fig. 2) and the expected magnitude difference $\Delta m_{12}$ between the two lensed images 1 and 2 of a distant quasar (see Fig. 3). The values of the impact parameters $b_{\Delta m i}(i=1-4)$ and $b_{\max }$ also depend on the angular distances $D_{O Q}, D_{O L}$, and $D_{L Q}$ between the observer $O$, the quasar $Q$ and the lens $L$; they are easily derived numerically in the framework of the point mass 
TABLE 4. Results on $F$ and $N^{\text {exp }}$ from our statistical GL studies (see text).

\begin{tabular}{llccrr}
\hline \hline$\Omega_{0}$ & $\lambda_{0}$ & $F_{\text {lo }}(99.7 \%)$ & $F_{\text {up }}(99.7 \%)$ & \multicolumn{1}{c}{$N_{\text {lo }}^{\exp }$} & $N_{\text {up }}^{\text {exp }}$ \\
\hline 1 & 0 & 0.005 & 0.478 & 1.3 & 2.6 \\
0.1 & 0.9 & 0.001 & 0.090 & 6.7 & 13.7 \\
0 & 1 & 0.0004 & 0.037 & 16.7 & 33.4 \\
0 & 0 & 0.003 & 0.265 & 2.3 & 4.7 \\
\hline \hline
\end{tabular}

lens model (cf. Claeskens 1992; see also Nemiroff 1991a, b and Kassiola et al. 1991).

One may then write the relation between the effective optical depth $\tau$ for the lensing of $N_{Q}$ quasars by a cosmic population of compact lenses, with mass $\mathscr{M}_{L}$ and density parameter $\Omega_{L}$, as follows:

$$
\begin{aligned}
\tau & =N_{L} / N_{Q} \\
& =\Omega_{L} \sum_{q=1}^{N_{Q}} G\left(z_{q}, b_{q}, \text { Type, } \theta_{\text {max }}, \mathscr{M}_{L}, \Omega_{L}\right) S_{\text {cat }} / N_{Q},
\end{aligned}
$$

where most of the quantities have been defined in Sec. 3.1 [cf. Eq. (1)] and where

$$
\begin{aligned}
G\left(z_{q}, b_{q}, \text { Type, } \theta_{\max }, \mathscr{M}_{L}, \Omega_{L}\right) \\
=\frac{3 H_{0} c}{8 G \mathscr{M}_{L}} \cdot \int_{0}^{z_{q}} \frac{(1+z)}{\sqrt{1+\Omega_{0} z}}\left[\left[\operatorname{Min}\left(b_{\Delta m 2}^{2}, b_{\max }^{2}\right)\right.\right. \\
\left.\quad-\operatorname{Min}\left(b_{\Delta m 1}^{2}, b_{\max }^{2}\right)\right] B\left(b_{q}, \Delta_{\min }\right) \\
\quad+\left[\operatorname{Min}\left(b_{\Delta m 4}^{2}, b_{\max }^{2}\right)-\operatorname{Min}\left(b_{\Delta m 3}^{2}, b_{\max }^{2}\right)\right] \\
\left.\quad \times B\left(b_{q}, \Delta_{\min }\right)\right] d z .
\end{aligned}
$$

In the above expression, the magnification bias $B\left(b_{q}, \Delta_{\text {min }}\right)$ is calculated by means of Eq. (5) adopting the compact lens (CL) point source amplification probability

$P_{\mathrm{CL}}(\Delta)=\frac{e^{\Delta / 1.086}\left(e^{2 \Delta / 1.086}-1\right)^{-1.5}}{1.086\left[0.5\left(10^{\left(\Delta m_{i / i+1}\right) / 5}+10^{-\left(\Delta m_{i / i+1}\right) / 5}\right)-1\right]}$,

with

$$
\Delta_{\min }=2.5 \log \left[\frac{10^{\left(\Delta m_{i / i+1}\right) / 2.5}+1}{10^{\left(\Delta m_{i / i+1}\right) / 2.5}-1}\right],
$$

where $\Delta m_{i / i+1}$ (for $i=1$ or $i=3$ ) represents a typical value of the ASF between the intersecting solutions $\theta_{\Delta m i}$ and $\theta_{\Delta m i+1}$ (see Fig. 3). Let us note that, in Eq. (11), $G$ appears to be a function of $\Omega_{L}$ because of the slight dependence of the Dyer-Roeder angular distance $D_{L Q}$ on $\Omega_{L}$ (Dyer \& Roeder 1973). In practice, Eq. (11) is successfully solved numerically by means of a Gauss-Legendre method with 9 points.

\subsubsection{Numerical applications and results}

Making use of the 469 quasars observed within the ESO-KP, Crampton et al., Yee et al., and HST snapshot samples, we have applied Eqs. (10)-(13) in order to set
TABLE 5. Results on $\Omega_{L}$ from our statistical GL studies (see text).

\begin{tabular}{llcc}
\hline \hline$\Omega_{0}$ & $\lambda_{0}$ & $\Omega_{L}^{\text {sup }}(99.7 \%)$ & $\mathscr{M}_{L}\left(\mathscr{M}_{\odot}\right)$ \\
\hline 1 & 0 & 0.057 & $10^{10}$ \\
1 & 0 & 0.021 & $5 \times 10^{10}$ \\
1 & 0 & 0.018 & $10^{11}$ \\
1 & 0 & 0.028 & $5 \times 10^{11}$ \\
1 & 0 & 0.059 & $10^{12}$ \\
0.1 & 0.9 & 0.005 & $10^{11}$ \\
0.0 & 1.0 & 0.003 & $10^{11}$ \\
0.0 & 0.0 & 0.012 & $10^{11}$ \\
\hline \hline
\end{tabular}

upper limits on the parameter $\Omega_{L}$ for compact lenses in the mass range $10^{10}-10^{12} \mathscr{M}_{\odot}$ (see Table 5). With a $99.7 \%$ confidence level and adopting $H_{0}=50 \mathrm{~km} / \mathrm{s} / \mathrm{Mpc}, \Omega_{0}=1$ and $\Lambda=0$, we find that $\Omega_{L} \leqslant 0.02$; it is even likely that $\Omega_{L} \ll 0.02$ (if none of the known GL systems is due to a compact deflector).

\subsubsection{Discussion}

In 1982, Canizares used the small observed variation in the equivalent widths of broad emission lines recorded in the spectrum of quasars to exclude a closure density of compact objects in the range $10^{-2} \leqslant \mathscr{M}_{L} / \mathscr{M}_{\odot} \leqslant 10^{5}$.

From the number of multiply imaged radio sources detected in the VLA lens survey, Hewitt (1986) could derive,under reasonable assumptions, $\Omega_{L}<0.4$ for compact objects in the mass range $10^{11}-10^{13} \mathscr{M}_{\odot}$.

Using the results reported by Crampton et al. (1989) for the number of possible GL candidates identified in a sample of HLQs (see Sec. 2.2), Nemiroff (1991a) has applied his "detection volume formalism" to deduce that $\Omega_{L}<1$ (respectively, $\Omega_{L}<0.25$ ) for compact objects having a mass $\mathscr{M}_{L}>10^{9.9} \mathscr{M}_{\odot}$ (respectively, $\mathscr{M}_{L}$ $\left.>10^{10.3} \mathscr{M}_{\odot}\right)$. Note, however, that Nemiroff did not include in his calculations the-important-magnification bias correction factor, discussed previously.

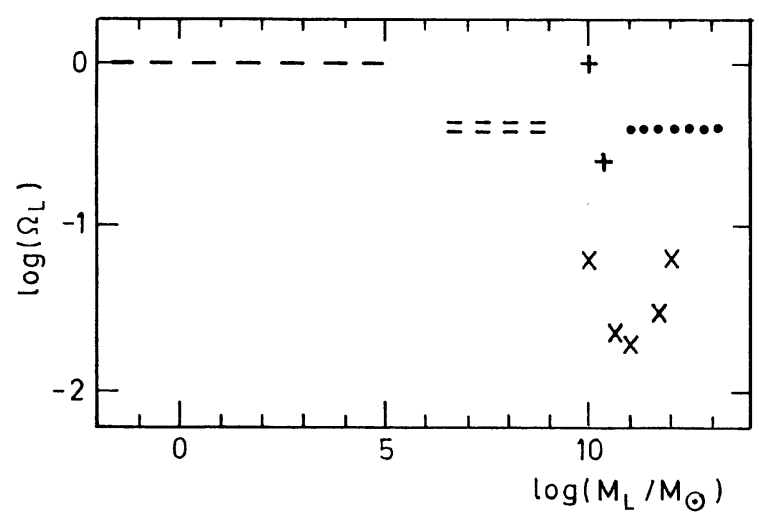

FIG. 4. Adopting $H_{0}=50 \mathrm{~km} / \mathrm{s} / \mathrm{Mpc}, \Omega_{0}=1$ and $\Lambda=0$, we have illustrated in this diagram the various upper bounds derived for the density parameter $\Omega_{L}$ of compact lenses, with mass $\mathscr{M}_{L}$, capable of producing multiple images of distant sources. The various determinations are from (-) Canizares (1982), (.) Hewitt (1986), (+) Nemiroff (1991a), (=) Kassiola et al. (1991) and $(X)$ the present work. 


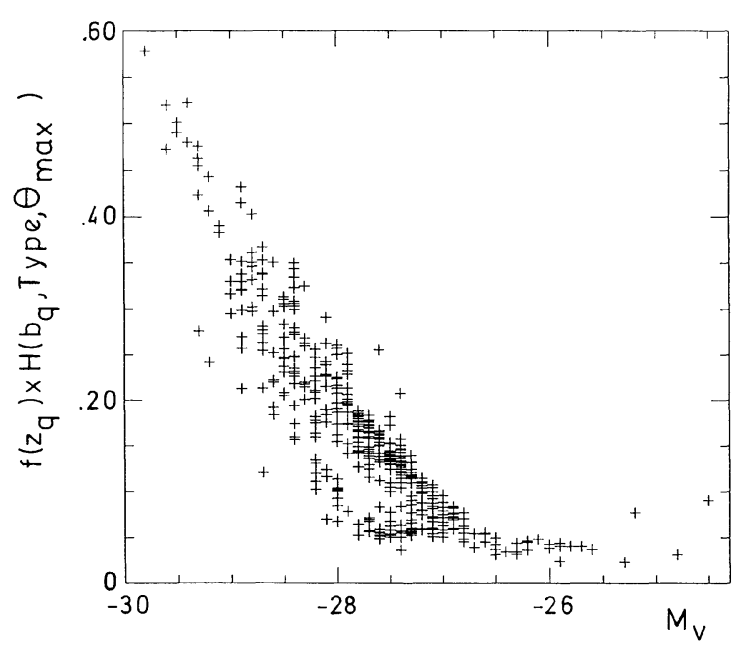

FIG. 5. The quantity $f\left(z_{q}\right) H\left(b_{q}\right.$, Type, $\left.\theta_{\max }\right)$ is represented as a function of the absolute magnitude $M_{V}$ for each of the 469 HLQs observed in the merged sample.

Kassiola et al. (1991) have investigated the constraints that can be imposed on the cosmic density of uniformly distributed intergalactic compact objects in the range $10^{7} \leqslant \mathscr{M}_{L} / \mathscr{M}_{\odot} \leqslant 10^{9}$ using VLBI observations of compact radio sources. They exclude $\Omega_{L}>0.4$ at the $99.7 \%$ confidence level.

We have summarized in Fig. 4 all previous estimates quoted for $\Omega_{L}$ over a wide range of deflector masses. It is striking that the cosmological density of compact objects in the mass range $10^{10}-10^{12} \mathscr{M}_{\odot}$ is presently the best constrained one: it already appears to be much lower than that of known galaxies!

\section{DISCUSSION}

\subsection{Quasars Multiply Imaged by Singular Isothermal Sphere (SIS) Galaxies}

Considering first the astronomical aspect of gravitational lensing, it is obvious from Eq. (6) that in order to maximize the detection of GL systems among HLQs, one should select quasars such that the quantity $f\left(z_{q}\right) H\left(b_{q}\right.$, Type, $\left.\theta_{\max }\right)$ is as large as possible. We have represented in Fig. 5, for each of the 469 HLQs belonging to the merged sample, this quantity as a function of the absolute magnitude $M_{V}$. The correlation existing between the quantity $f\left(z_{q}\right) H\left(b_{q}\right.$,Type, $\left.\theta_{\max }\right)$ and $M_{V}$ clearly shows why selecting a sample of quasars with an intrinsic brightness as high as possible constitutes the best approach to search for new lenses as well as to constrain most efficiently the value of the effectiveness parameter $F$.

Addressing now the observational strategy to follow when searching for new GLs, we see from Eq. (6) that the function $H\left(b_{q}\right.$, Type, $\left.\theta_{\max }\right)$ should also be maximized with respect to the ASF, i.e., one should make use of the instruments characterized by the best resolving power (e.g., under optimal seeing conditions for the ground based observations) with as large a dynamical range as possible. We have illustrated in Fig. 6 the histogram of that quantity for

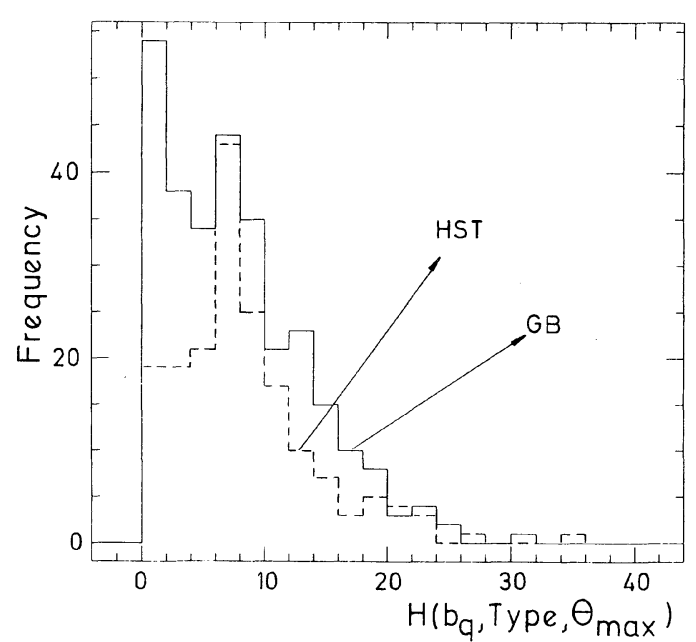

FIG. 6. Histogram of the function $H\left(b_{q}\right.$, Type, $\left.\theta_{\max }\right)$ [see Eqs. (6) and (7)] for the 659 observations of HLQs either obtained with ground based (GB) telescopes or with HST.

the 659 observations of the 469 HLQs available so far, in accordance with the "angular selection functions" given in Table 2 (see also Fig. 2). Although the angular resolution of the HST observations is substantially superior to that of the ground based data, the corresponding dynamical range is presently more limited, resulting in a very comparable efficiency for the detection of GL candidates. Given the lower costs of operating instruments from the ground, there is no doubt that observations of HLQs obtained with a 2-4 $\mathrm{m}$ ground based telescope and characterized by a large dynamical range ( $\Delta m \geqslant 5 \mathrm{mag})$ and high angular resolution (optimal seeing conditions or using a speckle camera) should provide in the near future the best strategy to search for new GLs.

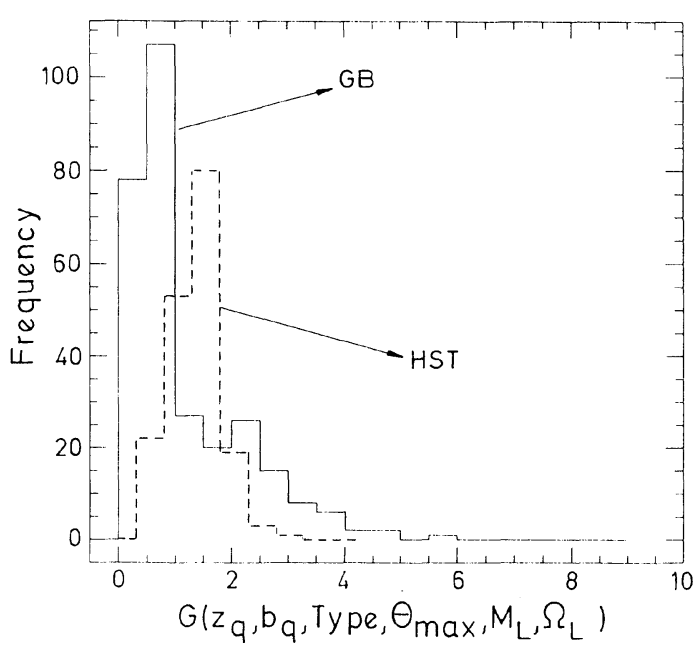

FIG. 7. Histogram of the quantity $G\left(z_{q}, b_{q}\right.$, Type, $\left.\theta_{\max }, \mathscr{M}_{L}, \Omega_{L}\right)$ [see Eqs. (10) and (11)] for the 659 observations of HLQs either obtained with ground based (GB) telescopes or with $H S T$ ( $\mathscr{M}_{L}$ $\left.=10^{10} \mathscr{M}_{\odot}\right)$. 


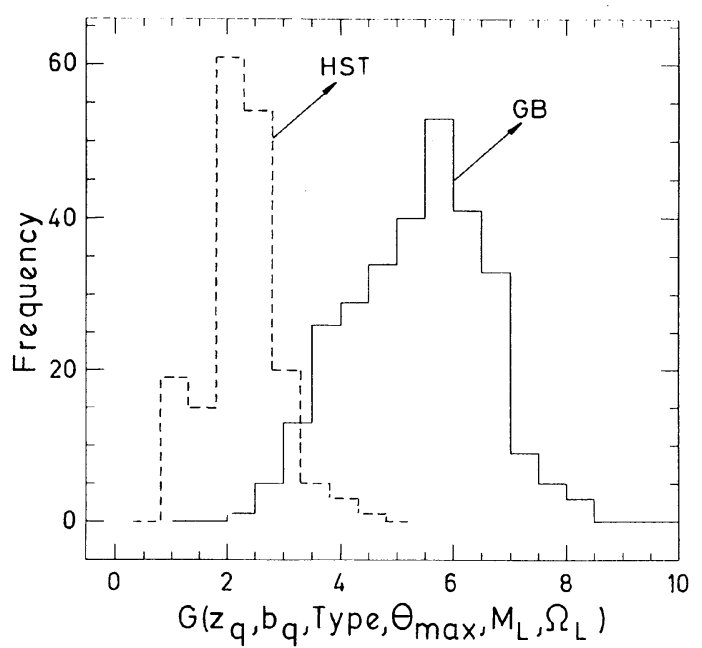

FIG. 8. Histogram of the quantity $G\left(z_{q}, b_{q}\right.$ Type, $\left.\theta_{\max } \mathscr{M}_{L}, \Omega_{L}\right)$ [see Eqs. (10) and (11)] for the 659 observations of HLQs either obtained with ground based (GB) telescopes or with $H S T$ ( $\mathscr{M}_{L}$ $=10^{11} \mathscr{M}_{\odot}$ ).

\subsection{Quasars Multiply Imaged by Compact Objects}

We have represented in Figs. 7 and 8 the histograms of the quantity $G\left(z_{q}, b_{q}\right.$, Type, $\left.\theta_{\max }, \mathscr{M}_{L}, \Omega_{L}\right)$ appearing in Eqs. (10)-(11) for $\mathscr{M}_{L}=10^{10}$ and $\mathscr{M}_{L}=10^{11} \mathscr{M}_{\odot}$, respectively. We conclude here also that the efficiency of ground based instruments used to constrain the values of the density parameter $\Omega_{L}$ in the mass range $5 \times 10^{10}-10^{12} \mathscr{M}_{\odot}$ appears to be as good as that of $H S T$. Note that only for $\mathscr{M}_{L} \leqslant 5 \times 10^{10} \mathscr{M}_{\odot}$, does the efficiency of $H S T$ become superior to that of ground based observations. Replacement of the WFPC I camera by WFPC II should, of course, improve much the efficiency of future $H S T$ observations.

\section{GENERAL CONCLUSIONS}

Generally speaking, gravitational lensing effects may alter the observed number counts of distant quasars in various ways. Whereas we have considered in this work the phenomenon of multiply imaged quasars at $\simeq 0.1^{\prime \prime}-3^{\prime \prime}$ angular scales, it should be noted that magnification of distant quasars may also result from microlensing effects due to stellar and/or planetarylike objects in intervening galaxies as well as by matter (galaxies, clusters, large sheets of dust and gas, other-unknown?-forms of dark matter, etc.) at various possible locations along the line-of-sight.

In the present paper, we have applied statistical gravitational lens studies to a sample of 469 highly luminous quasars (HLQs), either observed from the ground or with $H S T$, in order to estimate the effectiveness parameter $F$ of singular isothermal sphere (SIS) galaxies as well as upper bounds on the density parameter $\Omega_{L}$ of compact objects in the mass range $10^{10}-10^{12} \mathscr{M}_{\odot}$. For the values of the cosmological parameters $H_{0}=50 \mathrm{~km} / \mathrm{s} / \mathrm{Mpc}, \Omega_{0}=1$ and $\Lambda=0$, we have found at a significance level of $99.7 \%$ that (i) $0.005<F<0.478$, a result which overlaps current expectations (FT) and that (ii) $\Omega_{L} \leqslant 0.02$, implying that less than $2 \%$ of the closure density of the Universe is in the form of compact objects with masses in the range $10^{10} \leqslant \mathscr{M}_{L} / \mathscr{M}_{\odot} \leqslant 10^{12}$.

We have reviewed in Sec. 3.1.3 all the types of uncertainties which may affect the previous estimates of $F$, and we have concluded that it is presently hazardous to use the observed fraction of multiply lensed quasars in order to derive an estimate of the cosmological parameter $\Lambda$. Note that our estimated upper bounds on $\Omega_{L}$ also strongly depend on the adopted value for $\Lambda$ (see Table 5 and FFK).

In Sec. 4, we have compared the efficiencies of ground based and of HST observations carried out for the 469 selected HLQs in a search for new lenses. We have concluded that these efficiencies were presently very comparable (cf. Sec. 4.2). Furthermore, because of the present difficulties related to the spherical aberration of $H S T$, to the variation of the PSF over the field of the WF/PC camera, etc. and due to a significant difference in operational costs, we have argued that obtaining direct imagery of HLQs, with a 2-4 m class ground based telescope equipped with an optical speckle camera or working under very good seeing conditions, still constitutes the best approach to search for multiply lensed quasars. Observing a medium size sample of HLQs with a speckle camera characterized by a dynamical range $\Delta m=5 \mathrm{mag}$ would allow one to constrain the density parameter $\Omega_{L}$ to less than 0.01 in the mass range $10^{8}-10^{10} \mathscr{M}_{\odot}$, a search for massive compact objects near $10^{9} \mathscr{M}_{\odot}$ being of course dictated by the possible existence of dark black holes with masses similar to AGN central engines. Note that quite a number of HLQs observed with $H S T$ show possible structures at $0.1^{\prime \prime}-0.2^{\prime \prime}$ scales (cf. Maoz et al. 1992b); however, because of the complex PSF shape, it is not possible to conclude at present whether these structures are real or due to PSF artifacts.

As may be seen from Fig. 4, much work has still to be done in order to improve the constraints on $\Omega_{L}$ for compact objects in the mass range $10^{-8}-10^{10} \mathscr{M}_{\odot}$. It is likely that novel techniques will be developed and applied in the near future. For instance, Refsdal (1990) has suggested to use simultaneous observations of remote quasars from the Earth and from a distant spacecraft in order to search for lensing effects by compact lenses in the mass range $10^{-8}$ $10^{4} \mathscr{M}_{\odot}$. Kassiola et al. (1991) have predicted that using the capabilities of the VLBA $\left(\theta_{\text {res }}=0.0005^{\prime \prime}\right.$, dynamical range $R=1000$ ), future observations should allow one to exclude or discover $\Omega_{L} \geqslant 10^{-3}$ for compact objects in the range $10^{5} \leqslant \mathscr{M}_{L} / \mathscr{M}_{\odot} \leqslant 10^{8}$. The optical interferometric mode of future Very Large Telescopes (e.g., the ESO VLT) should also allow one to probe quite soon the mass range $10^{6}-10^{8} \mathscr{M}_{\odot}$ for hypothetical compact objects.

In the context of observational searches for massive astrophysical compact objects located in the halo of our Galaxy (MACHOs, cf. Paczynski 1986), let us mention that ongoing projects should lead to the possibility of exploring the presence of compact deflectors in the mass range $10^{-8}$ $10^{-1} \mathscr{M}_{\odot}$, including brown dwarf and planet candidates (Bennett et al. 1991; Alcock et al. 1992; Vidal-Madjar et al. 1992; etc.). 
J. S. wishes to thank Chris Kochanek for having encouraged us to publish the present data and results. Our thanks go also to Jean-Pierre Swings and Philippe Véron for reading the manuscript and for their comments, and to Armand Kransvelt for the preparation of the figures. Part of this research has been supported by Contract No. ARC 90/94-140 "Action de Recherche Concertée de la Communauté Française" (Belgium) and a FNRS grant (D.H.). A.V.F. acknowledges the support of NSF Grant No. AST8957063.

\section{REFERENCES}

Alcock, C., et al. 1992, in the Proceedings of the Hamburg Conference on Gravitational Lenses, Lecture Notes in Physics 406, 156, edited by $R$. Kayser, T. Schramm, and L. Nieser (Springer, Berlin)

Bahcall, J. N., Maoz, D., Doxsey, R., Schneider, D. P., Bahcall, N. A., Lahav, O., \& Yanny, B. 1992a, ApJ, 387, 56

Bahcall, J. N., Maoz, D., Schneider, D. P., Yanny, B., \& Doxsey, R. 1992b, ApJ, 392, L1

Bennett, D. P., et al. 1991, preprint

Blandford, R. D., \& Narayan, R. 1992, ARA\&A, 30, 311

Boyle, B. J., Shanks, T., \& Peterson, B. A. 1988, MNRAS, 235, 935

Canizares, C. R. 1982, ApJ, 263, 508

Claeskens, J. F. 1992, Master thesis, Liège University

Crampton, D., McClure, R. D., Fletcher, J. M., \& Hutchings, J. B. 1989 AJ, 98, 1188

Crampton, D., McClure, R. D., \& Fletcher, J. M. 1992, preprint

Djorgovski, S., Perley, R., Meylan, G., \& McCarthy, P. 1987, ApJ, 321, L17

Djorgovski, S., \& Meylan, G. 1989a, in Active Galactic Nuclei, IAU Symposium No. 134, edited by D. Osterbrock and J. Miller (Kluwer, Dordrecht), p. 269

Djorgovski, S., \& Meylan, G. 1989b, in Gravitational Lenses, edited by J. M. Moran et al. (Springer, New York), p. 173

Dyer, C. C., \& Roeder, R. C. 1973, ApJ, 180, L31

Fukugita, M., \& Turner, E. L. 1991, MNRAS, 253, 99 (FT)

Fukugita, M., Futamase, T., \& Kasai, M. 1990, MNRAS, 246, 24P (FFK)

Fukugita, M., Futamase, T., Kasai, M., \& Turner, E. L. 1992, ApJ, 393, 3

Hartwick, F. D. A., \& Schade, D. 1990, ARA\&A, 28, 437

Hewitt, J. N. 1986, Ph.D. thesis, MIT

Jaroszynski, M. 1991, MNRAS, 249, 430

Kassiola, A., Kovner, I., \& Blandford, R. 1991, ApJ, 381, 6

Kochanek, C. 1991a, private communication

Kochanek, C. S. 1991b, ApJ, 379, 517

Kochanek, C. S. 1991c, preprint

Magain, P., Surdej, J., Swings, J. P., Borgeest, U., Kayser, R., Kühr, H., Refsdal, S., \& Remy, M. 1988, Nature, 334, 325

Magain, P., Remy, M., Surdej, J., Swings, J. P., \& Smette, A. 1990, in Lecture Notes in Physics, Gravitational Lensing, Proceedings of the Toulouse Workshop on Gravitational Lenses, September 1989, edited by Y. Mellier, B. Fort, and G. Soucail (Springer, Berlin), Vol. 360, 88

Magain, P., Hutsemékers, D., Surdej, J., \& Van Drom, E. 1992a, in the Proceedings of the Hamburg Conference on Gravitational Lenses, Lecture Notes in Physics 406, edited by R. Kayser, T. Schramm, and L. Nieser (Springer, Berlin), p. 88

Magain, P., Surdej, J., Vanderriest, C., Pirenne, B., \& Hutsemékers, D. 1992b, A\&A, 253, L13

Mao, S. 1991, ApJ, 380, 9

Maoz, D., et al. 1992a, ApJ, 386, L1

Maoz D., Bahcall, J. N., Doxsey, R., Schneider, D. P., Bahcall, N. A., Lahav, O., \& Yanny, B. 1992b, preprint

Meylan, G., \& Djorgovski, S. 1989, ApJ, 338, L1

Narayan, R. 1989, ApJ, 339, L53
Nemiroff, R. J. 1989, ApJ, 341, 579

Nemiroff, R. 1991a, Phys. Rev. Lett., 66, 538

Nemiroff, R. 1991b, Comments Astrophys., 15, 139

Paczynski, B. 1986, ApJ, 304, 1

Pirenne, B., Surdej, J., \& Magain, P. 1992, ST-ECF Newsletter 17, 22

Press, W. H., \& Gunn, J. E. 1973, ApJ, 185, 397

Refsdal, S. 1990, review paper in Lecture Notes in Physics, Gravitational

Lensing, Proceedings of the Toulouse Workshop on Gravitational

Lenses, September 1989, edited by Y. Mellier, B. Fort, and G. Soucail (Springer, Berlin), Vol. 360, p. 13

Schneider, P. 1991, MPA preprint 584

Surdej, J., et al. 1987, Nature 329, 695

Surdej, J., et al. 1988a, A\&A, 198, 49

Surdej, J., Magain, P., Swings, J. P., Remy, M., Borgeest, U., Kayser, R., Refsdal, S., \& Kühr, H. 1988b, invited paper to the First D.A.E.C. workshop on Large Scale Structures, proceedings edited by C. Balkowski and S. Gordon, p. 95

Surdej, J., et al. 1988c, Astron. Soc. of the Pac. Conf. Series 2, 183

Surdej, J., et al. 1989, The Messenger, 55, 8

Surdej, J. 1990, review paper in Lecture Notes in Physics, Gravitational Lensing, Proceedings of the Toulouse Workshop on Gravitational Lenses, September 1989, edited by Y. Mellier, B. Fort, and G. Soucail (Springer, Berlin), Vol. 360, pp. 57, 311

Surdej, J., et al. 1992a, invited paper to the Second D.A.E.C. meeting on the Distribution of Matter in the Universe, edited by G. A. Mamon and D. Gerbal, p. 97

Surdej, J., Claeskens, J. F., Hutsemékers, D., Magain, P., \& Pirenne, B. $1992 b$, in the Proceedings of the Hamburg Conference on Gravitational Lenses, Lecture Notes in Physics 406, edited by R. Kayser, T. Schramm, and L. Nieser (Springer, Berlin), p. 27

Surdej, J. et al. 1993, in preparation

Swings, J. P., Magain, P., Remy, M., Surdej, J., Smette, A., Hutsemékers, D., \& Van Drom, E. 1990, in Lecture Notes in Physics, Gravitational Lensing, Proceedings of the Toulouse Workshop on Gravitational Lenses, September 1989, edited by Y. Mellier, B. Fort, and G. Soucail (Springer, Berlin), Vol. 360, p. 83

Turner, E. L. 1990, ApJ, 365, L43

Turner, E. L., Ostriker, J. P., \& Gott III, J. R. 1984, ApJ, 284, 1 (TOG) Van Drom, E. 1992, in the Proceedings of the Hamburg Conference on Gravitational Lenses, Lecture Notes in Physics 406, edited by R. Kayser, T. Schramm, and L. Nieser (Springer, Berlin), p. 250

Van Drom, E., Surdej, J., Hutsemékers, D., Magain, P., Gosset, E., Shaver, P., \& Melnick, J. 1993, A\&A (submitted)

Véron-Cetty, M. P., \& Véron, P. 1987, ESO Scientific Report No. 5 Véron-Cetty, M. P., \& Véron, P. 1991, ESO Scientific Report No. 10 Vidal-Madjar, A., Aubourg, E., Kunth, D., Ferlet, R., \& Mochkovitch, R. et al. 1992, invited paper to the Second D.A.E.C. Workshop on Large Scale Structures in the Universe (in press)

Wallington, S., \& Narayan, R. 1992, preprint

Weymann, R. J., Latham, D., Angel, J. R. P., Green, R. F., Liebert, J. W., Turnshek, D. A., Turnshek, D. E., \& Tyson, J. A. 1980, Nature 285,641

Yee, H. K. C., Filippenko, A. V., \& Tang, D. 1993, AJ, 105, 7 